\title{
WEAK AND STRONG LIMIT VALUES
}

\author{
EVGENY A. POLETSKY
}

\begin{abstract}
The classical results about the boundary values of holomorphic or harmonic functions on a domain $D$ state that under additional integrability assumptions these functions have limits along specific sets approaching boundary. The proofs of these results are based on properties of smooth boundaries used to define the approach regions and on estimates of representing kernels along these regions.

This paper attempts to look at the situation when no assumptions about the boundary smoothness are made and, consequently, no natural definitions of approach regions could be given.
\end{abstract}

\section{INTRODUCTION}

The theory of the boundary values of functions defined on a domain $D \subset \mathbb{R}^{n}$ and lying in some class $\mathcal{S}$ tries to answer the following questions:

(1) Is there a trace operator $\mathcal{T}$ mapping $\mathcal{S}$ into some class $\mathcal{S}^{b}$ of functions on $\partial D$ so that $\mathcal{T} u$ is neatly associated with $u$ ?

(2) Is there a restoring operator $\mathcal{R}$ mapping $\mathcal{S}^{b} \oplus\{$ some information $\}$ so that $\mathcal{T} \mathcal{R}$ is an identity and $\mathcal{R} \mathcal{T}$ is the identity provided that "some information" is supplied?

The most developed boundary values theory is for subclasses of (sub)harmonic functions. For bounded domains the classical Perron-Wiener-Brelot (PWB) method $($ see $[\mathrm{H}])$ provides harmonic solutions to the second problem for the class of functions integrable with respect to the harmonic measure, while the Fatou-Naïm-Doob Theorem (see $[\mathrm{AG}]$ ) asserts the existence of minimal fine limits of non-negative harmonic functions almost everywhere on the boundary. However, no connections between the latter limits and the given function were established for general domains.

When $n=2$ and $D$ is the unit disk the work of P. Fatou and F. Riesz completely answered these questions when $\mathcal{S}$ is a Hardy space either of harmonic functions or holomorphic functions. It was shown that for a function $h$ in such a space non-tangential limits exist almost everywhere at the boundary with respect to the surface length and play the role of the trace $\mathcal{T} h$. The Poisson integral plays the role of $\mathcal{R}$. J. E. Littlewood expanded this work to the class of subharmonic functions with harmonic majorants using the Laplacian as "some information" and the Riesz decomposition formula as $\mathcal{R}$.

In 1936 I. I. Privalov and P. K. Kuznetsov lifted Littlewood's results to $n=3$ and $D$ be a ball. E. D. Solomentsev in $[\mathrm{S}]$ generalized their work to any $n$ and $D$ be a $C^{2}$-domain. In [Da] B. E. J. Dahlberg expanded this result to $C^{1,1}$-domains.

2000 Mathematics Subject Classification. Primary: 28A33;secondary: 30E25, 31E20.

Key words and phrases. boundary values.

The author was supported by the NSF Grant DMS-0900877. 
D. S. Jerison and C. E. Kenig continued this work in [JK1] and for a larger class of $L_{1}^{p}$-domains showed that under an integrability condition on the boundary data $\phi$ the PWB solution is given by the Poisson formula and has non-tangential limits equal to $\phi$ almost everywhere on the boundary. They also proved that bounded harmonic functions on such domains have non-tangential limits almost everywhere at the boundary with respect to the surface area.

In [JK2] D. S. Jerison and C. E. Kenig generalized their results to non-tangentially accessible domains. However, the two major changes of the classical theory were made in this paper. First of all, the surface area was abandoned and replaced by the harmonic measure $\mu$ and, secondly, the Hardy spaces were defined as sets of functions for which the maximal non-tangential function belongs to $L^{p}(\mu)$. This approach was mostly used in recent publications.

Similar results for subharmonic functions seem to be non-existent. Moreover, in [Da] B. E. J. Dahlberg showed that there are a $C^{1, \alpha}$-domain $D \subset \mathbb{R}^{2}(0<\alpha<1)$ and a negative subharmonic function on $D$ which has no limits along normals on a set of positive length. So the validity of the the Riesz decomposition formula was not clear.

The situation was even worse for plurisubharmonic functions, where the LelongJensen formula (see [D]) replaces the Riesz decomposition formula. Roughly speaking, to get good boundary values results one, firstly, establishes them on compact subdomains and then exhausts $D$ by open sets $D_{j} \subset \subset D, j=1,2, \ldots$ If the boundary is nice the exhaustion can be made even nicer. The formulas representing functions at $x \in D$ (e.g., Riesz or Lelong-Jensen formulas) involve an integral of the function over the boundary $S_{j}=\partial D_{j}$ over some measure $\mu_{x j}$ and an integral over $D_{j}$.

While it is easy to establish that the volume integrals converge to similar integrals as $j \rightarrow \infty$, the surface integrals present the major difficulty. In the classical potential theory the measures $\mu_{x j}$ are the surface areas times the Poisson kernel. The behavior of the Poisson kernel on smooth domains is well studied and it allows to prove that the surface integrals converge to similar integrals of pre-existing boundary values.

However, an attempt to replicate this for subharmonic functions on non-smooth domains or plurisubharmonic functions on hyperconvex domains fails due to the absence of knowledge about the measures $\mu_{x j}$. In the case of plurisubharmonic functions except of $[\mathrm{D}]$ only $[\mathrm{BPT}]$ addresses the latter problem for strongly convex domains with smooth boundary.

In this paper we suggest an approach which, firstly, abandons the non-tangential limits and, secondly, returns to the classical definition of Hardy spaces. To understand the situation we start with the general problem of different type convergence for sequences $\left\{\phi_{j} \mu_{j}\right\}$, where $\mu_{j}$ is a measure on $S_{j}$. Standard integrability conditions show that the sequence $\left\{\phi_{j} \mu_{j}\right\}$ has a subsequence converging weak-* to a measure $\phi_{*} \mu_{x}$ and we restrict our attention to weak-* converging sequences. The functions $\phi_{*}$ can be considered as the weak limit values. The main disadvantage of these functions $\phi_{*}$ is their bad correlation with products. It is not true, in general, that $(\phi \psi)_{*}=\phi_{*} \psi_{*}$ - the identity one needs to prove the integral formulas (see examples in Section 2).

However, we establish that if the sequence $\left\{\left|\phi_{j}\right|^{p} \mu_{j}\right\}$ also has the weak-* limit $\nu$, then $\nu \geq\left|\phi_{*}\right|^{p} \mu$. Surprisingly, $\nu=\left|\phi_{*}\right|^{p} \mu$ if and only if $\phi_{*}$ has much stronger 
properties and we call it the strong limit values and denote by $\phi^{*}$ (for the precise definition see Section 3. This theory is developed in Sections 2 and 3. In particular, we show that if $\psi_{*}$ exists then $\phi^{*} \psi_{*}=(\phi \psi)_{*}$.

In Section 4 we address the question when the strong limit values exist or when $\nu=\left|\phi_{*}\right|^{p} \mu$. We consider a space of functions with the weak limit values and satisfying an integral inequality (5). For example, if the space in question is the space of subharmonic functions, then the inequality is the classical estimate of the value of a function $\phi$ at some point through the convolution of $\phi_{*}$ and the Poisson kernel.

Under mild conditions on the kernel we prove that the functions in such spaces have the strong limit values. The section also contains several results showing when these mild conditions hold.

In Section 5 we look at functions defined on $D$ and define boundary values as the strong limit values for all possible sequences of exhaustions. We give some sufficient conditions for functions to have boundary values, establish a general result allowing to expand integral representations and show that functions which have classical radial limits also have boundary values in our sense.

Section 6 contains applications of exposed theory to harmonic and subharmonic functions on a general regular domain $D$. We introduce the Hardy spaces $\mathcal{S}^{p}(D)$ of (sub)harmonic functions on $D$ and show that any function in $L^{p}\left(\partial D, \mu_{x_{0}}\right)$ is the trace of a function in $\mathcal{S}^{p}(D)$ and the Poisson integral serves as restoring operator. We also prove that the Riesz decomposition formula is valid for a function $u \in$ $\mathcal{S}^{p}(D)$ if and only if $u$ has the boundary values.

The last section 7 contains the expansion of Lelong-Jensen formula to $L^{p}$-classes of plurisubharmonic functions on strongly pseudoconvex domains and the polydisk. This case was also studied in [CK].

The author is grateful to Michael Stessin for his strong encouragement to write this paper and to the referee whose suggestions improved the exposition.

\section{WEAK LIMIT VALUES}

Let $K$ be a compact metric space, and let $M=\left\{\mu_{j}\right\}$ be a sequence of regular Borel measures on $K$ converging weak-* in $C^{*}(K)$ to a finite measure $\mu$. We denote the set $\operatorname{supp} \mu_{j}$ by $K_{j}$ and $\operatorname{supp} \mu$ by $K_{0}$. Let $\phi=\left\{\phi_{j}\right\}$ be a sequence of Borel functions $\phi_{j}$ on $K_{j}$. We let

$$
\|\phi\|_{L^{p}(M)}=\limsup _{j \rightarrow \infty}\left\|\phi_{j}\right\|_{L^{p}\left(K_{j}, \mu_{j}\right)} .
$$

In general, the weak- $*$ limit of measures $\phi_{j} \mu_{j}$ need not to be absolutely continuous with respect to $\mu$. But as the following lemma shows this is the case when $\|\phi\|_{L^{p}(M)}<\infty$ for some $p>1$.

Lemma 2.1. If $\|\phi\|_{L^{p}(M)} \leq A, p>1$, and the measures $\phi_{j} \mu_{j}$ converge weak-* to a measure $\mu^{\prime}$ on $K$, then there is a function $\phi_{*} \in L^{p}\left(K_{0}, \mu\right)$ such that $\mu^{\prime}=\phi_{*} \mu$ and $\left\|\phi_{*}\right\|_{L^{p}\left(K_{0}, \mu\right)} \leq A$.

Proof. First of all, we show that $\operatorname{supp} \mu^{\prime}=\operatorname{supp} \mu$, i.e., for any $h \in C(K)$

$$
\mu^{\prime}(h)=\int_{K} h d \mu^{\prime}=\int_{K_{0}} h d \mu^{\prime} .
$$


For this for $\delta>0$ we take the closed $\delta$-neighborhood $V$ and the open $2 \delta$-neighborhood $W$ of $K_{0}$. Let $f_{1}$ be a non-negative continuous function on $K$ taking values between 0 and 1 , which is equal to 1 on $V$ and whose support lies in $W$. Let $f_{2}=1-f_{1}$. Then

$$
\lim _{j \rightarrow \infty} \int_{K_{j}} f_{2} h d \mu_{j}=\int_{K_{0}} f_{2} h d \mu=0 .
$$

By Hölder's inequality

$$
\left|\int_{K_{j}} f_{2} h \phi_{j} d \mu_{j}\right| \leq\left\|\phi_{j}\right\|_{L^{p}\left(K_{j}, \mu_{j}\right)}\left(\int_{K_{j}} f_{2} h d \mu_{j}\right)^{1 / q},
$$

where $1 / p+1 / q=1$. Thus

$$
\mu^{\prime}(h)=\mu^{\prime}\left(f_{2} h\right)+\mu^{\prime}\left(f_{1} h\right)=\lim _{j \rightarrow \infty} \int_{K} f_{2} h \phi_{j} d \mu_{j}+\mu^{\prime}\left(f_{1} h\right)=\mu^{\prime}\left(f_{1} h\right) .
$$

Hence for every open neighborhood $Y$ of $K_{0}$

$$
\mu^{\prime}(h)=\int_{Y} h d \mu^{\prime}
$$

and this implies that

$$
\mu^{\prime}(h)=\int_{K} h d \mu^{\prime}=\int_{K_{0}} h d \mu^{\prime} .
$$

By Hölder's inequality

$$
\left|\int_{K_{j}} h \phi_{j} d \mu_{j}\right| \leq\left\|\phi_{j}\right\|_{L^{p}\left(K_{j}, \mu_{j}\right)}\|h\|_{L^{q}\left(K_{j}, \mu_{j}\right)} .
$$

Hence

$$
\left|\int_{K} h d \mu^{\prime}\right|=\lim _{j \rightarrow \infty}\left|\int_{K_{j}} h \phi_{j} d \mu_{j}\right| \leq A\|h\|_{L^{q}\left(K_{0}, \mu\right)} .
$$

So the functional

$$
\mu^{\prime}(h)=\int_{K} h d \mu^{\prime}=\int_{K_{0}} h d \mu^{\prime}
$$

on $C(K)$ admits an unique extension to a continuous functional on $L^{q}\left(K_{0}, \mu\right)$. Thus there is a function $\phi_{*} \in L^{p}\left(K_{0}, \mu\right)$ such that $\left\|\phi_{*}\right\|_{L^{p}\left(K_{0}, \mu\right)} \leq A$ and

$$
\int_{K_{0}} h d \mu^{\prime}=\int_{K_{0}} h \phi_{*} d \mu
$$

for any $h \in C(K)$. Therefore, $\mu^{\prime}=\phi_{*} \mu$.

If the measures $\left\{\phi_{j} \mu_{j}\right\}$ converge weak- $*$ to a measure $\phi_{*} \mu$, then the function $\phi_{*}$ will be called the weak limit values of $\phi$. We will denote by $\mathcal{A}(M)$ the space of all sequences $\phi$ of Borel functions $\phi_{j}$ on $K_{j}$ which have weak limit values and by $\mathcal{A}^{p}(M)$ those sequences $\phi$ in $\mathcal{A}(M)$ for $\|\phi\|_{L^{p}(M)}<\infty$. 
While the weak-* convergence of measures $\left\{\phi_{j} \mu_{j}\right\}$ frequently occurs it does not correlate well with algebraic operations. First of all, as the following example shows, it does not imply the weak-* convergence of the sequence $\phi^{p}=\left\{\left|\phi_{j}\right|^{p} \mu_{j}\right\}$.

Example: Let $K=K_{0}=[0,1]$ and all measures $\mu_{j}$ be equal to the Lebesgue measure $\mu$ on $[0,1]$. For each $j$ we split $[0,1]$ into intervals $I_{j k}=\{x: k / j \leq x<$ $(k-1) / j\}, k=0, \ldots, j-1$. When $j$ is even we let $\phi_{j}(x)=0$ if $x \in I_{j k}$ and $k$ is even and we let $\phi_{j}(x)=2$ if $x \in I_{j k}$ and $k$ is odd. When $j$ is odd then we let $\phi_{j}(x)=1$ for all $x \in[0,1]$. Then the measures $\phi_{j} \mu_{j}$ converge weak- $*$ to $\mu$, while the measures $\phi_{j}^{2} \mu_{j}$ do not converge weak-*. For even $j$ they converge to $2 \mu$ and for odd ones to $\mu$.

Even if the sequence $\phi^{p}=\left\{\left|\phi_{j}\right|^{p} \mu_{j}\right\}$ weak limit values it is not true that $\left|\phi_{*}\right|^{p}=$ $\left(\left|\phi^{p}\right|\right)_{*}$ as the example below shows.

Example: We take measures and intervals from the previous example and for all $j$ we let $\phi_{j}(x)=a>0$ if $x \in I_{j k}$ and $k$ is even and we let $\phi_{j}(x)=b>0$ if $x \in I_{j k}$ and $k$ is odd. Then the measures $\phi_{j} \mu_{j}$ converge weak-* to $\phi_{*} \mu$, where $\phi_{*} \equiv(a+b) / 2$, while the measures $\phi_{j}^{2} \mu_{j}$ converge weak- $*$ to $\nu=\left(a^{2}+b^{2}\right) \mu / 2$.

Note that $\phi_{*}^{2} \mu \leq \nu$ and the theorem below shows that this crucial observation is always true.

Theorem 2.2. If $\phi \in \mathcal{A}^{p}(M), p>1$, and the measures $\left|\phi_{j}\right|^{p} \mu_{j}$ converge weak-* to a measure $\nu$ on $K$, then $\nu \geq\left|\phi_{*}\right|^{p} \mu$.

Proof. Let $A=\left\|\phi_{*}\right\|_{L^{p}(K, \mu)}$ and let $G(a, b)=\left\{x \in K_{0}: a \leq \phi_{*}(x)<b\right\}$. Suppose that $b>a>0$ and let $X \subset G(a, b)$ be a Borel set. Suppose that $\mu(X)=m>0$. We fix $\varepsilon>0$, such that $a \mu(X)-A \varepsilon^{1 / q}>0,1 / p+1 / q=1$, and take an open set $O$ and a closed set $C$ such that $C \subset X \subset O, a \mu(C)-A \varepsilon^{1 / q}>0, \nu(O \backslash C)<\varepsilon$ and $\mu(O \backslash C)<\varepsilon$. Let $f$ be a continuous function on $K$ equal to 0 on $K \backslash O$, equal to 1 on $C$ and taking values between 0 and 1 elsewhere.

Then

$$
\begin{aligned}
\nu(O) & \geq \int_{K} f d \nu=\lim _{j \rightarrow \infty} \int_{K} f\left|\phi_{j}\right|^{p} d \mu_{j} \geq \lim _{j \rightarrow \infty}\left|\int_{K} f \phi_{j} d \mu_{j}\right|^{p}\left(\int_{K} f d \mu_{j}\right)^{1-p} \\
& =\left|\int_{K} f \phi_{*} d \mu\right|^{1-p}\left(\int_{K} f d \mu\right)^{1-p} .
\end{aligned}
$$

Now

$$
\int_{K} f \phi_{*} d \mu=\int_{C} f \phi_{*} d \mu+\int_{O \backslash C} f \phi_{*} d \mu \geq a \mu(C)+\int_{O \backslash C} f \phi_{*} d \mu
$$

and

$$
\left|\int_{O \backslash C} f \phi_{*} d \mu\right| \leq\left\|\phi_{*}\right\|_{L^{p}(K, \mu)}\|f\|_{L^{q}(K, \mu)} \leq A \varepsilon^{1 / q}
$$

Hence

$$
\left|\int_{K} f \phi_{*} d \mu\right|^{p} \geq\left(a \mu(C)-A \varepsilon^{1 / q}\right)^{p}
$$


Thus

$$
\nu(O) \geq\left(a \mu(C)-A \varepsilon^{1 / q}\right)^{p} \mu^{1-p}(C) .
$$

Letting $\varepsilon$ go to 0 we see that $\nu(X) \geq a^{p} \mu(X)$. Hence

$$
\int_{X} \phi_{*}^{p} d \mu \leq b^{p} \mu(X) \leq \frac{b^{p}}{a^{p}} \nu(X) .
$$

If $b<a<0$ then replacing $\phi$ by $-\phi$ we see that

$$
\int_{X}\left|\phi_{*}\right|^{p} d \mu \leq|b|^{p} \mu(X) \leq\left|\frac{b}{a}\right|^{p} \nu(X) .
$$

Note that if $\mu(X)=0$ both inequalities above are trivially true.

If $X$ is any Borel set in $K_{0} \cap\left\{\phi_{*} \geq 0\right\}$, we denote by $X_{\varepsilon}, \varepsilon>0$, the intersection of $X$ and the set $\left\{\phi_{*}>\varepsilon\right\}$, where $\varepsilon>0$. Let us take some $\delta>0$, form a sequence $a_{0}=\varepsilon, a_{k+1}=(1+\delta)^{1 / p} a_{k}$ and let $X_{\varepsilon}^{k}=X_{\varepsilon} \cap G\left(a_{k}, a_{k+1}\right)$. Then

$$
\int_{X_{\varepsilon}} \phi_{*}^{p} d \mu=\sum_{k=0}^{\infty} \int_{X_{\varepsilon}^{k}} \phi_{*}^{p} d \mu \leq(1+\delta) \nu\left(X_{\varepsilon}\right) .
$$

Thus

$$
\int_{X_{\varepsilon}} \phi_{*}^{p} d \mu \leq \nu(X)
$$

If $X_{\varepsilon}^{\prime}=X \backslash X_{\varepsilon}$ then

$$
\int_{X_{\varepsilon}^{\prime}} \phi_{*}^{p} d \mu \leq \varepsilon^{p} \mu(X)
$$

Thus

$$
\int_{X} \phi_{*}^{p} d \mu \leq \nu(X)+\varepsilon^{p} \mu(X)
$$

and it shows that

$$
\int_{X} \phi_{*}^{p} d \mu \leq \nu(X) .
$$

If $X \subset \operatorname{supp} \mu \cap\left\{\phi_{*}<0\right\}$ then a consideration of the function $-\phi$ shows that

$$
\int_{X}\left|\phi_{*}\right|^{p} d \mu \leq \nu(X) .
$$

Thus $\nu \geq\left|\phi_{*}\right|^{p} \mu$.

We finish this section with a couple of technical lemmas.

Lemma 2.3. Let $\left\{\mu_{j}\right\}$ be a sequence of regular Borel measures on $K$ converging weak-* to $\mu$ and let $A$ be a closed set in $K$. Then for every $\varepsilon>0$ there is an open set $O$ containing $A$ and $j_{0}$ such that $\mu_{j}(O)<\mu(A)+\varepsilon$ when $j \geq j_{0}$. 
Proof. Let us fix some $\delta>0$ and $0<a<1$ whose precise values will be determined later and take a continuous function $f$ on $K$ which is equal to 1 on $A, 0 \leq f<1$ elsewhere and $\mu(f)<\mu(A)+\delta$. Then there is $j_{0}$ such that $\mu_{j}(f)<\mu(f)+\delta$ when $j \geq j_{0}$. Since $\mu_{j}(f) \geq a \mu_{j}(\{f>a\})$ we see that

$$
\mu_{j}(\{f>a\})<\frac{\mu(f)+\delta}{a}<\frac{\mu(A)+2 \delta}{a} .
$$

If we take $a$ and $\delta$ satisfying the inequality $2 \delta<a \varepsilon+(a-1) \mu(A)$ and let $O=\{f>a\}$ then $\mu_{j}(O)<\mu(A)+\varepsilon$.

This lemma, in general, does not hold for non-closed sets. For example, let $\mu$ be the Lebesgue measure on $[0,1]$ and

$$
\mu_{j}=\frac{1}{n} \sum_{k=0}^{n} \delta_{k / j}
$$

Then $\mu_{j}$ converge weak-* to $\mu, \mu(\mathbb{Q})=0$ but $\mu_{j}(\mathbb{Q}) \geq 1$.

To resolve this problem we introduce the regular weak-* convergence. Namely, we say that measures $\left\{\mu_{j}\right\}$ converge weak- $*$ to $\mu$ regularly if they converge weak-* and for any $\mu$-measurable set $A$ in $K_{0}=\operatorname{supp} \mu$ and every $\varepsilon>0$ there is an open set $O$ containing $A$ and $j_{0}$ such that $\mu_{j}(O)<\mu(A)+\varepsilon$ when $j \geq j_{0}$.

Usually, it is easy to verify the regularity of a weak-* convergence. If not then the lemma below gives a criterion which is especially useful for boundary values.

Lemma 2.4. Suppose that in assumptions of Lemma 2.3 $K_{j} \cap K_{0}=\emptyset$ for all $j$. Then the measures $\left\{\mu_{j}\right\}$ converge weak-* to $\mu$ regularly.

Proof. We already know that the lemma holds for all closed set. Let $A \subset K_{0}$ be a relatively open set. We look at the distance function $d(x)=\operatorname{dist}(x, \partial A)$ on $K_{0}$ and note that $\mu(\{d(x)=a\})>0$ only for countably many values of $a$. Choosing a sequence of $a_{k} \searrow 0$ such that $\mu\left(\left\{d(x)=a_{k}\right\}\right)=0$ we let $A_{k}=\left\{x \in A: a_{k} \leq\right.$ $\left.d(x) \leq a_{k+1}\right\}$. Then the sets $A_{k}$ are closed, $A=\cup A_{k}$ and $\mu(A)=\sum \mu\left(A_{k}\right)$.

For $\varepsilon>0$ and any $k$ we find $j_{k}$ and an open set $O_{k}$ such that $A_{k} \subset O_{k}$ and $\mu_{j}\left(O_{k}\right)<\mu\left(A_{k}\right)+2^{-k} \varepsilon$ when $j>j_{k}$. Let $O_{k}^{\prime}=O_{k} \backslash \cup_{j=1}^{j_{k}} K_{j}$. Then the sets $O_{k}^{\prime}$ are open, contain $A_{k}$ and $\mu_{j}\left(O_{k}^{\prime}\right)<\mu\left(A_{k}\right)+2^{-k} \varepsilon$ for all $j$. If $O=\cup O_{k}^{\prime}$ then $\mu_{j}(O)<\mu(A)+\varepsilon$ for all $j$.

If $A$ is any $\mu$-measurable set in supp $\mu$ then we find a relatively open set $A^{\prime} \subset K_{0}$ such that $\mu\left(A^{\prime}\right)<\mu(A)+\varepsilon / 2$ and then an open set $O$ in $K$ containing $A^{\prime}$ such that $\mu_{j}(O)<\mu\left(A^{\prime}\right)+\varepsilon / 2$ for all $j$. Then $\mu_{j}(O)<\mu(A)+\varepsilon$.

\section{Strong Limit values}

We will need a more precise Hölder's inequality. For $x \geq 0$ and $p>1$ let

$$
\Phi_{p}(x)=x^{p}-p(x-1)-1 .
$$

Clearly, $\Phi_{p}(x) \geq 0$ and $\Phi_{p}(x)=0$ if and only if $x=1$.

If $p \geq 2$ then $\Phi_{p}(x) \geq|x-1|^{p}$. Indeed, if $x>1$ then differentiating both sides we come to an evident inequality

$$
x^{p-1}-(x-1)^{p-1} \geq 1 .
$$

If $0 \leq x \leq 1$, then the same trick leads to another evident inequality

$$
x^{p-1}+(1-x)^{p-1} \leq 1 \text {. }
$$


Let

$$
\bar{f}=f_{K} f d \mu=\frac{1}{\mu(K)} \int_{K} f d \mu .
$$

Replacing $x$ with $f / \bar{f}$ in (1) and integrating both sides we get the following proposition.

Proposition 3.1. Let $(K, \mu)$ be a measure space, $0<\bar{\mu}=\mu(K)<\infty$, and let $f$ be a non-negative measurable function on $K$ with $\|f\|_{L^{1}(K, \mu)}<\infty$. If $p>1$ then

$$
\left(f_{K} f d \mu\right)^{-p} f_{K} f^{p} d \mu=1+f_{K} \Phi_{p}\left(\frac{f}{\bar{f}}\right) d \mu .
$$

If $p \geq 2$ then

$$
\left(f_{K} f d \mu\right)^{-p} f_{K} f^{p} d \mu \geq 1+f_{K}|f / \bar{f}-1|^{p} d \mu .
$$

We will need the following immediate consequence of this proposition.

Lemma 3.2. In assumptions of Proposition 3.1 for $p>1$ and $c>0$ there is a constant $\alpha(p, c)$ such that if the left side of (2) is smaller than $1+\varepsilon, \varepsilon>0$, then $\mu(\{|f / \bar{f}-1|>1+c\})<\alpha(p, c) \varepsilon \mu(K)$.

We say that a sequence $\phi \in \mathcal{A}(M)$ has the strong limit values on $K_{0}$ with respect to $M$ if there is a $\mu$-measurable function $\phi^{*}$ on $K_{0}$ such that for any $b>a$ and any $\varepsilon, \delta>0$ there is $j_{0}$ and an open set $O \subset K$ containing $G(a, b)=\left\{x \in K_{0}: a \leq\right.$ $\left.\phi^{*}(x)<b\right\}$ such that

$$
\mu_{j}\left(\left\{\phi_{j}<a-\varepsilon\right\} \cap O\right)+\mu_{j}\left(\left\{\phi_{j}>b+\varepsilon\right\} \cap O\right)<\delta
$$

when $j \geq j_{0}$. The function $\phi^{*}$ will be called the strong limit values of $\phi$.

Let us indicate some properties of strong limit values.

Theorem 3.3. Suppose that $\phi$ has the strong limit values on $K_{0}$ equal to $\phi^{*}$. Then:

(1) any two choices of $\phi^{*}$ coincide $\mu$-a.e., the sequences $c \phi$ and $|\phi|^{p}$ have strong limit values and $(c \phi)^{*}=c \phi^{*}$ and $\left(|\phi|^{p}\right)^{*}=\left|\phi^{*}\right|^{p}$;

(2) if the sequence $\left\{\mu_{j}\right\}$ converges weak-* regularly to $\mu$ and a sequence $\psi \in$ $\mathcal{A}(M)$ has the strong limit values $\psi^{*}$, then the sequences $\phi+\psi$ and $\phi \psi$ have the strong limit values and $(\phi+\psi)^{*}=\phi^{*}+\psi^{*}$ and $(\phi \psi)^{*}=\phi^{*} \psi^{*}$.

Proof. 1) Suppose that $\phi$ has two different strong limit values $\phi_{1}^{*}$ and $\phi_{2}^{*}$. Suppose that for some $\varepsilon>0$ there are $a<b$ and $c<d$ such that $c>b+2 \varepsilon$ and $\mu\left(\left\{a \leq \phi_{1}^{*}<\right.\right.$ $\left.b\} \cap\left\{c \leq \phi_{2}^{*}<d\right\}\right)=\alpha>0$. Let us take open sets $O_{1}$ containing $\left\{a \leq \phi_{1}^{*}<b\right\}$ and $\mathrm{O}_{2}$ containing $\left\{c \leq \phi_{2}^{*}<d\right\}$ such that

$$
\mu_{j}\left(\left\{\phi_{j}<a-\varepsilon\right\} \cap O_{1}\right)+\mu_{j}\left(\left\{\phi_{j}>b+\varepsilon\right\} \cap O_{1}\right)<\alpha / 4
$$

and

for large $j$.

$$
\mu_{j}\left(\left\{\phi_{j}<c-\varepsilon\right\} \cap O_{2}\right)+\mu_{j}\left(\left\{\phi_{j}>d+\varepsilon\right\} \cap O_{2}\right)<\alpha / 4
$$

If $O=O_{1} \cap O_{2}$ then $\mu_{j}(O)>\alpha / 2$ for large $j$. Hence

$$
\mu_{j}\left(\left\{c-\varepsilon \leq \phi_{j} \leq d+\varepsilon\right\} \cap O\right)>\alpha / 4
$$

and, consequently, $\mu_{j}\left(\left\{b+\varepsilon<\phi_{j}\right\} \cap O\right)>\alpha / 4$ and we get a contradiction. Hence, $\mu\left(\left\{a \leq \phi_{1}^{*}<b\right\} \cap\left\{c \leq \phi_{2}^{*}<d\right\}\right)=0$ when $c>b+2 \varepsilon$. 
Since $\varepsilon>0$ is arbitrary we see that $\mu\left(\left\{a \leq \phi_{1}^{*}<b\right\} \cap\left\{c \leq \phi_{2}^{*}<d\right\}\right)=0$ when $c>b$. Thus $\phi_{2}^{*} \leq b \mu$-a.e. on $\left\{a \leq \phi_{1}^{*}<b\right\}$ and this shows that $\phi_{2}^{*} \leq \phi_{1}^{*} \mu$-a.e. By symmetry, $\phi_{2}^{*} \leq \phi_{1}^{*} \mu$-a.e.

The facts that $(c \phi)^{*}=c \phi^{*}$ and $\left(|\phi|^{p}\right)^{*}=\left|\phi^{*}\right|^{p}$ are trivial.

2) We will prove only that $(\phi+\psi)^{*}=\phi^{*}+\psi^{*}$. Other claims can be proved similarly.

For given numbers $\varepsilon, \delta>0$ we find $c<d$, such that $\mu(X)<\delta / 2$, where

$$
X=\left\{\phi^{*}<c\right\} \cup\left\{\psi^{*}<c\right\} \cup\left\{\phi^{*}>d\right\} \cup\left\{\psi^{*}>d\right\} .
$$

Then we split the interval $[c, d]$ into consecutive intervals $\left[a_{k}, a_{k+1}\right]$ such that $a_{k+1}-$ $a_{k} \leq \varepsilon, 0 \leq k \leq n$.

If $a \leq \phi^{*}+\psi^{*}<b$ and $a_{k} \leq \phi^{*}<a_{k+1}$, then $a-a_{k+1}<\psi^{*}<b-a_{k}$. Hence the set $G(a, b)=\left\{a \leq \phi^{*}<b\right\}$ lies in the set

$$
X \cup\left(\bigcup_{k=0}^{n-1}\left(\left\{a_{k} \leq \phi^{*}<a_{k+1}\right\} \cap\left\{a-a_{k+1}<\psi^{*}<b-a_{k}\right\}\right)\right) .
$$

Let $O_{k}^{\prime}$ be open sets containing $\left\{a_{k} \leq \phi^{*}<a_{k+1}\right\}$ and such that

$$
\mu_{j}\left(\left\{\phi_{j}<a_{k}-\varepsilon\right\} \cap O_{k}^{\prime}\right)+\mu_{j}\left(\left\{\phi_{j}>a_{k+1}+\varepsilon\right\} \cap O_{k}^{\prime}\right)<\frac{\delta}{n},
$$

while $O_{k}^{\prime \prime}$ be open sets containing $\left\{a-a_{k+1}<\psi^{*}<b-a_{k}\right\}$ and such that

$$
\mu_{j}\left(\left\{\psi_{j}<a-a_{k+1}-\varepsilon\right\} \cap O_{k}^{\prime \prime}\right)+\mu_{j}\left(\left\{\psi_{j}>b-a_{k}+\varepsilon\right\} \cap O_{k}^{\prime \prime}\right)<\frac{\delta}{n}
$$

when $j$ is large. Let $O^{\prime \prime \prime}$ be an open set containing $X$ and such that $\mu_{j}\left(O^{\prime \prime \prime}\right)<\delta$ for large $j$. We let $O_{k}=O_{k}^{\prime} \cup O_{k}^{\prime \prime}$ and $O=O^{\prime \prime \prime} \cup \cup_{k=0}^{n-1} O_{k}$. Clearly, $G(a, b) \subset O$.

If $x \in O_{k} \cap K_{j}, a_{k}-\varepsilon \leq \phi_{j}(x)<a_{k+1}+\varepsilon$ and $a-a_{k+1}-\varepsilon \leq \psi_{j}(x) \leq b-a_{k}+\varepsilon$, then $a-2 \varepsilon<\phi_{j}(x)+\psi_{j}(x)<b+2 \varepsilon$. Thus the measure $\mu_{j}$ of those $x \in O_{k} \cap K_{j}$ for which $\phi_{j}(x)+\psi_{j}(x)<a-2 \varepsilon$ or $\phi_{j}(x)+\psi_{j}(x)>b+2 \varepsilon$ does not exceed $2 \delta / n$. Consequently,

$$
\mu_{j}\left(\left\{\phi_{j}+\psi_{j}<a-2 \varepsilon\right\} \cap O\right)+\mu_{j}\left(\left\{\phi_{j}+\psi_{j}>b+2 \varepsilon\right\} \cap O\right)<3 \delta .
$$

The next theorem provides a convenient criterion for the existence of limit values.

Theorem 3.4. If $\phi$ has the strong limit values $\phi^{*}$ on $K$ then for every $\varepsilon, \delta>0$ there is a function $f \in C(K)$ such that $\mu\left(\left\{\left|f-\phi^{*}\right|>\varepsilon\right\}\right)<\delta$ and $\mu_{j}\left(\left\{\left|f-\phi_{j}\right|>\varepsilon\right\}\right)<\delta$ for large $j$.

Moreover, if $\|\phi\|_{L^{p}(M)}<\infty, p \geq 1$, then for every $\varepsilon>0$ the function $f$ can be chosen so that $\|f\|_{L^{p}\left(\mu_{j}\right)}<\|\phi\|_{L^{p}(M)}+\varepsilon$ for large $j$ if $p<\infty$ and $\|f\| \leq$ $\|\phi\|_{L^{\infty}(M)}+\varepsilon$ if $p=\infty$.

If, additionally, the measures $\left\{\mu_{j}\right\}$ converge weak-* to $\mu$ regularly and for a sequence $\phi \in L^{p}(M)$ and a $\mu$-measurable function $\psi$ on $K_{0}$ and for every $\varepsilon, \delta>0$ there is a sequence $f$ with strong limit values $f^{*}$ such that $\mu\left(\left\{\left|f^{*}-\psi\right|>\varepsilon\right\}\right)<\delta$ and $\mu_{j}\left(\left\{\left|f_{j}-\phi_{j}\right|>\varepsilon\right\}\right)<\delta$ for large $j$, then $\phi$ has the strong limit values $\phi^{*}=\psi$ on $K$.

Proof. Suppose that $\phi$ has the strong limit values $\phi^{*}$ on $K$. For any $\delta>0$ we can find $b>0$ such that $\mu\left(\left\{\phi^{*}>b\right\}\right)+\mu\left(\left\{\phi^{*}<-b\right\}\right)<\delta$. Let $X=\{x \in \operatorname{supp} \mu$ : $\left.-b \leq \phi^{*}(x) \leq b\right\}$. We fix $\varepsilon>0$ and cover the interval $[-b, b]$ with intervals with 
end points $a_{k}=-b+k \varepsilon, 0 \leq k \leq[2 b / \varepsilon]+1=n$ and for each $k$ take a closed set $X_{k} \subset G\left(a_{k-1}, a_{k}\right)$ such that $\sum \mu\left(X_{k}\right)>\mu(X)-\delta$. Then we select disjoint open sets $O_{k}^{\prime}$ containing $X_{k}$ such that (3) holds for the given $\varepsilon$ and $c_{k} \delta / n$, where $c_{k}=\min \left\{1,\left|a_{k}\right|^{-p}\right\}$.

The next step is to pick up continuous functions $f_{k}$ on $K$ equal to 0 on $K \backslash O_{k}^{\prime}$, equal to $a_{k}$ on $X_{k}$ and taking values between 0 and $a_{k}$ elsewhere. Let $f=\sum f_{k}$, $O_{k}=\left\{\left|f_{k}-a_{k}\right|<\varepsilon\right\}$ and $O=\cup O_{k}$. Clearly, $\mu\left(\left\{\left|f-\phi^{*}\right|>2 \varepsilon\right\}\right)<3 \delta$.

Note that $\mu_{j}(K)<\mu(K)+\delta$ for large $j$. Also for large $j$ we have

$$
\mu_{j}(O)=\mu_{j}\left(\cup O_{k}\right)>\mu\left(\cup X_{k}\right)-\delta>\mu(X)-2 \delta>\mu(K)-3 \delta>\mu_{j}(K)-4 \delta .
$$

Let $A_{j k}=\left\{x \in K_{j} \cap O_{k}:\left|f(x)-\phi_{j}(x)\right|>3 \varepsilon\right\}$. Since

$$
\left|f-\phi_{j}\right| \leq\left|f-a_{k}\right|+\left|\phi_{j}-a_{k}\right|
$$

and $\left|f-a_{k}\right|<\varepsilon$, we see that if $x \in A_{j k}$, then $\left|\phi_{j}(x)-a_{k}\right|>2 \varepsilon$, i.e., either $\phi_{j}(x)<$ $a_{k}-2 \varepsilon$ or $\phi_{j}(x)>a_{k}+2 \varepsilon=a_{k+1}+\varepsilon$. By the choice of the sets $O_{k}$, the measure $\mu_{j}\left(A_{j k}\right)<c_{k} \delta / n$. Hence, $\mu_{j}\left(\left\{\left|f-\phi_{j}\right|>3 \varepsilon\right\} \cap O\right\}<\delta$. But $\mu_{j}(O)>\mu_{j}(K)-4 \delta$. Hence $\mu_{j}(K \backslash O)<3 \delta$ and this shows that $\mu_{j}\left(\left\{\left|f-\phi_{j}\right|>3 \varepsilon\right\}\right\}<4 \delta$.

If $\|\phi\|_{L^{\infty}(M)}=A<\infty$, then the very construction of $f$ shows that uniform norm of $f$ does not exceed $A+\varepsilon$. If $\|\phi\|_{L^{p}(M)}=A<\infty, 1 \leq p<\infty$, then we note that

$$
\int_{K}|f|^{p} d \mu_{j}=\sum \int_{O_{k}^{\prime}}|f|^{p} d \mu_{j}
$$

and $|f| \leq\left|a_{k}\right|$ on $O_{k}^{\prime}$ while the measure of those $x \in O_{k}^{\prime} \cap K_{j}$, where $\phi_{j}(x)<a_{k}-\varepsilon$ or $\phi_{j}(x)>a_{k}+2 \varepsilon$, does not exceed $c_{k} \delta / n$ when $j$ is large. Hence the measure of the set $B_{k j}=\left\{x \in O_{k}^{\prime} \cap K_{j}:|f(x)|>\left|\phi_{j}(x)\right|+2 \varepsilon\right\}$, does not exceed $c_{k} \delta / n$. Thus

$$
\int_{O_{k}^{\prime}}|f|^{p} d \mu_{j} \leq \int_{O_{k}^{\prime}}\left(\left|\phi_{j}\right|+2 \varepsilon\right)^{p} d \mu_{j}+\left|a_{k}\right|^{p} c_{k} \delta / n \leq \int_{O_{k}^{\prime}}\left(\left|\phi_{j}\right|^{p}+2 \varepsilon\right) d \mu_{j}+\delta / n .
$$

Hence,

$$
\int_{K}|f|^{p} d \mu_{j} \leq \int_{K}\left(\left|\phi_{j}\right|+2 \varepsilon\right)^{p} d \mu_{j}+\delta .
$$

By Minkowski's inequality

$$
\int_{K}\left(\left|\phi_{j}\right|+2 \varepsilon\right)^{p} d \mu_{j} \leq\left(\left\|\phi_{j}\right\|_{L^{p}\left(K_{j}, \mu_{j}\right)}+2 \varepsilon \mu_{j}^{1 / p}(K)\right)^{p} .
$$

Taking $\varepsilon$ and $\delta$ sufficiently small we get the proof of the statement.

To prove the last statement for given $\varepsilon, \delta>0$ we find a sequence $f$ with strong limit values $f^{*}$ satisfying the conditions of the theorem. If $G(a, b)=\{a \leq \psi<b\}$ and $X=G(a, b) \backslash\left\{a-\varepsilon<f^{*}<b+\varepsilon\right\}$, then $\mu(X)<\delta$. By the regularity of the weak-* convergence we can take an open set $O^{\prime}$ such that $X \subset O^{\prime}$ and $\mu_{j}\left(O^{\prime}\right)<\delta$ for large $j$.

Let us take an open set $O^{\prime \prime}$ containing $\left\{a-\varepsilon<f^{*}<b+\varepsilon\right\}$ such that

$$
\mu_{j}\left(\left\{f_{j}<a-2 \varepsilon\right\} \cap O^{\prime \prime}\right)+\mu_{j}\left(\left\{f_{j}>b+2 \varepsilon\right\} \cap O^{\prime \prime}\right)<\delta .
$$

If $O=O^{\prime \prime} \cup O^{\prime}$, then $G(a, b) \subset O$ and it is easy to see that

$$
\mu_{j}\left(\left\{\phi_{j}<a-3 \varepsilon\right\} \cap O\right)+\mu_{j}\left(\left\{\phi_{j}>b+3 \varepsilon\right\} \cap O\right)<2 \delta .
$$


It is not true that the weak limit values are equal to the strong limit values even when both do exist. For example, let all $\mu_{j}$ be equal to the Lebesgue measure $\mu$ on $[0,1]$. Surround the points $k / j, 0 \leq k \leq j$, by intervals of equal size and of total length $1 / j$. Let $\phi_{j}$ be equal to $j$ on these intervals and 0 outside. Then $\phi^{*} \equiv 0$, while $\phi_{*} \equiv 1$.

However, under additional integrability assumptions, the strong limit values correlate in the right way with powers and products of sequences even when one of the factors has only the weak limit values as the following chain of results shows.

Theorem 3.5. Suppose that a sequence $\phi \in \mathcal{A}(M)$ has the strong limit values $\phi^{*}$.

(1) If $\|\phi\|_{L^{p}(M)}=A<\infty, p>1$, then $\left\|\phi^{*}\right\|_{L^{p}(K, \mu)} \leq A$ and for any $s$, $1 \leq s<p$, the measures $|\phi|^{s} \mu_{j}$ converge weak-* to $\left|\phi^{*}\right|^{s} \mu$.

(2) If $\|\phi\|_{L^{p}(M)}<\infty, p>1$, and a sequence $\psi \in \mathcal{A}^{s}(M)$, where $s>q$, $1 / p+1 / q=1$, then the sequence $\left\{\phi_{j} \psi_{j} \mu_{j}\right\}$ converges weak-* to $\phi^{*} \psi_{*} \mu$.

Proof. 1) Let us take a sequence of $\varepsilon_{n}, \delta_{n} \searrow 0$. For $\delta_{n}$ and $\varepsilon_{n}$ let $f_{n}$ be a function from Theorem 3.4 with $\left\|f_{n}\right\|_{L^{p}(M)} \leq A+\delta_{n}$. If $h \in C(K)$ then

$$
\int_{K} h f_{n} d \mu=\lim _{j \rightarrow \infty} \int_{K} h f_{n} d \mu_{j} \leq\left(A+\delta_{n}\right)\|h\|_{L^{q}(K, \mu)} .
$$

Hence $f_{n} \in L^{p}(K, \mu)$ and $\left\|f_{n}\right\|_{L^{p}(K, \mu)} \leq A+\delta_{n}$.

The non-negative functions $\left|f_{n}\right|^{p}$ converge in measure to $\left|\phi^{*}\right|^{p}$ and $\left\|f_{n}\right\|_{L^{p}(K, \mu)} \leq$ $A+\delta_{n}$. Hence, due to the uniform integrability, $\left\|\phi^{*}\right\|_{L^{p}(K, \mu)} \leq A$.

Let $K_{n j}=\left\{x \in K_{j}:\left|f_{n}(x)-\phi_{j}(x)\right|<\varepsilon_{n}\right\}$. Firstly, since $\mu_{j}\left(K \backslash K_{n j}\right)<\delta_{n}$ for large $j$,

$$
\limsup _{j \rightarrow \infty} \int_{K \backslash K_{n j}}\left|\phi_{j}\right|^{s} d \mu_{j} \leq A^{s / p} \delta_{n}^{1-s / p}
$$

and

$$
\lim _{j \rightarrow \infty} \int_{K \backslash K_{n j}}\left|f_{n}\right|^{s} d \mu_{j} \leq\left(A+\delta_{n}\right)^{s / p} \delta_{n}^{1-s / p} .
$$

Secondly, if $|x-y|<\varepsilon_{n}$ then $\left.|| x\right|^{s}-|y|^{s} \mid \leq s\left(|y|+\varepsilon_{n}\right)^{s-1} \varepsilon_{n}$.

If a function $h \in C(K),\|h\|=1$, then

$$
\begin{aligned}
& \left|\int_{K} h\left(\left|\phi_{j}\right|^{s}-\left|f_{n}\right|^{s}\right) d \mu_{j}\right|=\left|\int_{K_{n j}} h\left(\left|\phi_{j}\right|^{s}-\left|f_{n}\right|^{s}\right) d \mu_{j}+\int_{K \backslash K_{n j}} h\left(\left|\phi_{j}\right|^{s}-\left|f_{n}\right|^{s}\right) d \mu_{j}\right| \\
& \leq s\left\|\phi+\varepsilon_{n}\right\|_{L^{s}\left(K_{j}, \mu_{j}\right)}^{s-1} \mu_{j}^{1 / s}(K) \varepsilon_{n}+2\left(A+\delta_{n}\right)^{s / p} \delta_{n}^{1-s / p}=C_{n},
\end{aligned}
$$

where $C_{n} \rightarrow 0$ as $n \rightarrow \infty$. Since

$$
\lim _{j \rightarrow \infty} \int_{K} h\left|f_{n}\right|^{s} d \mu_{j}=\int_{K} h\left|f_{n}\right|^{s} d \mu,
$$

we see that

$$
\begin{aligned}
\int_{K} h\left|f_{n}\right|^{s} d \mu-2 C_{n} & \leq \liminf _{j \rightarrow \infty} \int_{K} h\left|\phi_{j}\right|^{s} d \mu_{j} \\
& \leq \limsup _{j \rightarrow \infty} \int_{K} h\left|\phi_{j}\right|^{s} d \mu_{j} \leq \int_{K} h\left|f_{n}\right|^{s} d \mu+2 C_{n}
\end{aligned}
$$


for large $n$.

Let $K_{n}=\left\{x \in K_{0}:\left|f_{n}(x)-\phi^{*}(x)\right|<\varepsilon_{n}\right\}$. Then estimates similar to the used above give that

$$
\left|\int_{K} h\left(\left|\phi^{*}\right|^{s}-\left|f_{n}\right|^{s}\right) d \mu\right| \leq B_{n},
$$

where $B_{n} \rightarrow 0$ as $n \rightarrow \infty$. This shows that

$$
\lim _{n \rightarrow \infty} \int_{K} h\left|f_{n}\right|^{s} d \mu=\int_{K} h\left|\phi^{*}\right|^{s} d \mu .
$$

Hence

$$
\lim _{j \rightarrow \infty} \int_{K} h\left|\phi_{j}\right|^{s} d \mu_{j}
$$

exists and is equal to

$$
\int_{K} h\left|\phi^{*}\right|^{s} d \mu
$$

Thus $\left(|\phi|^{s}\right)_{*}=\left|\phi^{*}\right|^{s}$.

2) The proof proceeds at the same style and with the same notation. As before

$$
\begin{aligned}
& \left|\int_{K} h \psi_{j}\left(\phi_{j}-f_{n}\right) d \mu_{j}\right|=\left|\int_{K_{n j}} h \psi_{j}\left(\phi_{j}-f_{n}\right) d \mu_{j}+\int_{K \backslash K_{n j}} h \psi_{j}\left(\phi_{j}-f_{n}\right) d \mu_{j}\right| \\
& \left.\leq \varepsilon_{n}\left\|\psi_{j}\right\|_{L^{1}\left(K, \mu_{j}\right.}\right)+\left\|\phi_{j}-f_{n}\right\|_{L^{p}\left(K, \mu_{j}\right)}\left(\int_{K \backslash K_{n j}}\left|\psi_{j}\right|^{q} d \mu_{j}\right)^{1 / q} .
\end{aligned}
$$

Note that

Since

$$
\left(\int_{K \backslash K_{n j}}\left|\psi_{j}\right|^{q} d \mu_{j}\right)^{1 / q} \leq\left\|\psi_{j}\right\|_{L^{s}\left(K, \mu_{j}\right)} \delta_{n}^{1 / q-1 / s} .
$$

we see that

$$
\lim _{j \rightarrow \infty} \int_{K} h \psi_{j} f_{n} d \mu_{j}=\int_{K} h f_{n} \psi_{*} d \mu,
$$

$$
\begin{aligned}
\int_{K} h f_{n} \psi_{*} d \mu-C_{n} & \leq \liminf _{j \rightarrow \infty} \int_{K} h \phi_{j} \psi_{j} d \mu_{j} \\
& \leq \limsup _{j \rightarrow \infty} \int_{K} h \phi_{j} \psi_{j} d \mu_{j} \leq \int_{K} h f_{n} \psi_{*} d \mu+C_{n}
\end{aligned}
$$

for large $n$ and $C_{n} \rightarrow 0$ as $n \rightarrow \infty$.

Estimates similar to the used above give that

$$
\left|\int_{K} h\left(\phi^{*} \psi_{*}-f_{n} \psi_{*}\right) d \mu\right| \leq B_{n},
$$


where $B_{n} \rightarrow 0$ as $n \rightarrow \infty$. This shows that

$$
\lim _{n \rightarrow \infty} \int_{K} h f_{n} \psi_{*} d \mu=\int_{K} h \phi^{*} \psi_{*} d \mu .
$$

Hence

$$
\lim _{j \rightarrow \infty} \int_{K} h \phi_{j} \psi_{j} d \mu_{j}
$$

exists and is equal to

$$
\int_{K} h \phi^{*} \psi_{*} d \mu
$$

Now we came to the main theorem of this section. It claims that a sequence has the strong limit values when the inequality proved in Theorem 2.2 turns into an equality.

Theorem 3.6. Let $\|\phi\|_{L^{p}(M)} \in \mathcal{A}^{p}(M)$ for some $p>1$, and the measures $\left\{\left|\phi_{j}\right|^{p} \mu_{j}\right\}$ converge weak-* to $\nu$. If

$$
\nu(K)=\int_{K}\left|\phi_{*}\right|^{p} d \mu
$$

then the sequence $\phi$ has the strong limit values equal to $\phi_{*}$.

Proof. By Theorem $2.2 \nu=\left|\phi_{*}\right|^{p} d \mu$.

Let $f^{+}=\max \{f, 0\}$ and $f^{-}=\max \{-f, 0\}$. Since the measures $\phi_{j}^{+} \mu_{j}, \phi_{j}^{+} \mu_{j}$, $\left(\phi_{j}^{+}\right)^{p} \mu_{j}$ and $\left(\phi_{j}^{-}\right)^{p} \mu_{j}$ are uniformly bounded, we can find a subsequence $j_{k}$ such that all these measures converge weak-*.

If the measures $\phi_{j_{k}}^{+} \mu_{j_{k}}$ converge weak-* to $\psi \mu$, then $\psi \geq 0$ and the measures $\phi_{j_{k}}^{-} \mu_{j_{k}}$ converge weak-* to $\left(\psi-\phi_{*}\right) \mu$. Hence $\psi-\phi_{*} \geq 0$.

If the measures $\left(\phi_{j_{k}}^{+}\right)^{p} \mu_{j_{k}}$ converge weak-* to $\nu_{1}$ and the measures $\left(\phi_{j_{k}}^{-}\right)^{p} \mu_{j_{k}}$ converge weak- $*$ to $\nu_{2}$, then $\nu_{1}+\nu_{2}=\nu$ and by Theorem $2.2 \nu_{1} \geq \psi^{p} \mu$ and $\nu_{2} \geq\left(\psi-\phi_{*}\right)^{p} \mu$. Hence

$$
\left|\phi_{*}\right|^{p} \mu=\nu \geq \psi^{p} \mu+\left(\psi-\phi_{*}\right)^{p} \mu .
$$

But the inequality $|x|^{p} \geq y^{p}+(y-x)^{p}$, where $y \geq 0$ and $y-x \geq 0$, has only solution $y=x$ when $x \geq 0$ or $y=0$ when $x<0$. Hence $\psi=\left(\phi_{*}\right)^{+}$and $\psi-\phi^{*}=\left(\phi_{*}\right)^{-}$.

It follows that the measures $\phi_{j}^{+} \mu_{j}, \phi_{j}^{-} \mu_{j},\left(\phi_{j}^{+}\right)^{p} \mu_{j}$ and $\left(\phi_{j}^{-}\right)^{p} \mu_{j}$ converge weak-* to $\left(\phi_{*}\right)^{+} \mu,\left(\phi_{*}\right)^{-} \mu,\left(\phi_{*}^{+}\right)^{p} \mu$ and $\left(\phi_{*}^{-}\right)^{p} \mu$ respectively. Therefore, it is sufficient to prove our theorem only when all $\phi_{j} \geq 0$.

Let us fix $0<\varepsilon<1$ and $0<\delta<\varepsilon / 4$. We take $b \geq a>0$ such that $b / a<1+\delta$ and the set $X=G(a, b)=\left\{x \in \operatorname{supp} \mu: a \leq \phi_{*}(x)<b\right\}$. Suppose that $\mu(X)>0$. Then we take an open set $O_{1} \subset K$ and a closed set $C \supset K$ such that $C \subset X \subset O_{1}$, $\mu(C)>0$ and $\mu\left(O_{1} \backslash C\right)<\delta \mu(X)$. We find a non-negative continuous function $f$ on $K$ equal to 0 on $K \backslash O_{1}$, equal to 1 on $C$, taking values between 0 and 1 elsewhere and such that $\mu(f)<(1+\delta) \mu(C)$,

$$
\int_{K} f \phi_{*}^{p} d \mu<b^{p}(1+\delta)
$$


and

$$
b(1+\delta)>f_{K} f \phi_{*} d \mu>a(1+\delta)^{-1} .
$$

Let $\mu^{\prime}=f \mu$ and $\mu_{j}^{\prime}=f \mu_{j}$. Since $\mu_{j}^{\prime}, \phi_{j} \mu_{j}^{\prime}$ and $\phi_{j}^{p} \mu_{j}^{\prime}$ converge weak-* to $\mu^{\prime}, \phi_{*} \mu^{\prime}$ and $\phi_{*}^{p} \mu^{\prime}$ respectively, there is $j_{0}$ such that $\mu_{j}^{\prime}(K)<(1+\delta) \mu(C)$,

$$
f_{K} \phi_{j}^{p} d \mu_{j}^{\prime}<b^{p}(1+\delta)
$$

and

$$
b(1+\delta)>f_{K} \phi_{j} d \mu_{j}^{\prime}=\bar{\phi}_{j}>a(1+\delta)^{-1}
$$

when $j \geq j_{0}$. Hence

$$
\left(f_{K} \phi_{j} d \mu_{j}^{\prime}\right)^{-p} f_{K} \phi_{j}^{p} d \mu_{j}^{\prime}<(1+\delta)^{1+p} b^{p} a^{-p} \leq(1+\delta)^{1+2 p} \leq 1+r \delta,
$$

where $r$ depends only on $p$.

If $\phi_{j} \geq(1+\varepsilon) b$ then

$$
\phi_{j} / \bar{\phi}_{j} \geq(1+\varepsilon)(1+\delta)^{-1}>1+\varepsilon / 2
$$

since $\delta<\varepsilon / 4$ and $0<\varepsilon<1$. If $\phi_{j} \leq(1-\varepsilon) a$ then $\phi_{j} / \bar{\phi}_{j} \leq 1-\varepsilon / 2$. By Lemma 3.2

$$
\begin{aligned}
\mu_{j}^{\prime}\left(\left\{\phi_{j} \geq\right.\right. & (1+\varepsilon) b\})+\mu_{j}^{\prime}\left(\left\{\phi_{j} \leq(1-\varepsilon) a\right\}\right) \\
& \leq \mu_{j}^{\prime}\left(\left\{\left|\phi_{j} / \bar{\phi}_{j}-1\right|>\varepsilon / 2\right\}\right) \leq \alpha(p, \varepsilon / 2) r \delta \mu_{j}^{\prime}(K) .
\end{aligned}
$$

Let $O_{2}=\{f>1-\delta\}$. Then $O_{2}$ is open, lies in $O_{1}$ and contains $C$. Moreover,

$$
\begin{aligned}
& \mu_{j}\left(O_{2} \cap\left\{\phi_{j} \geq(1+\varepsilon) b\right\}\right)+\mu_{j}\left(O_{2} \cap\left\{\phi_{j} \leq(1-\varepsilon) a\right\}\right) \\
& \leq(1-\delta)^{-1}\left(\mu_{j}^{\prime}\left(\left\{\phi_{j} \geq(1+\varepsilon) b\right\}\right)+\mu_{j}^{\prime}\left(\left\{\phi_{j} \leq(1-\varepsilon) a\right\}\right)\right) \\
& \leq 2 \alpha(p, \varepsilon / 2) r \delta \mu_{j}^{\prime}(K) \leq 4 \alpha(p, \varepsilon / 2) r \delta \mu_{j}(X) .
\end{aligned}
$$

Finally we use the regularity of the weak-* convergence to take an open set $O_{3}$ containing $X \backslash C$ such that $\mu_{j}\left(O_{3}\right)<\delta \mu(X)$ when $j$ is sufficiently large. Let $O=O_{2} \cup O_{3}$. Then

$$
\mu_{j}\left(\left\{\phi_{j}<(1-\varepsilon) a\right\} \cap O\right)+\mu_{j}\left(\left\{\phi_{j}>(1+\varepsilon) b\right\} \cap O\right)<(4 \alpha(p, \varepsilon / 2) r+1) \delta \mu(X) .
$$

Thus we have shown that for every $0<\varepsilon<1,0<\delta<\varepsilon / 4, b \geq a>0$ and the set $X=G(a, b), \mu(X)>0$, there are a constant $c(p, \varepsilon)>0$ and an open set $O$ containing $X$ such that

$$
\mu_{j}\left(\left\{\phi_{j}<(1-\varepsilon) a\right\} \cap O\right)+\mu_{j}\left(\left\{\phi_{j}>(1+\varepsilon) b\right\} \cap O\right)<c(p, \varepsilon) \delta \mu(X)
$$

provided $b / a<1+\delta$.

Now for every $\varepsilon, \delta>0,0<\varepsilon<1,0<\delta<\varepsilon / 4$, we will show the existence of open sets satisfying (3) when $0<a \leq b<1$. For this we, firstly, note that if $\mu(X)=0$, then the existence of an open set for which (3) holds for all $\varepsilon$ and $\delta$ follows from the regularity of the weak-* convergence. If $a>0$ and $\mu(X)>0$, then we define $a_{0}=a, a_{k}=(1+\delta / 2) a_{k-1}$ while $a_{k}<b$ and the last $a_{n}=b$. Let $X_{k}=X \cap G\left(a_{k-1}, a_{k}\right)$. If $\mu\left(X_{k}\right)=0$ then we cover it by an open set $O_{k}$ such that $\mu_{j}\left(O_{k}\right)<\delta / n$ and if $\mu\left(X_{k}\right)>0$ then we cover it by an open set $O_{k}$ such that

$$
\mu_{j}\left(\left\{\phi_{j}<(1-\varepsilon) a_{k-1}\right\} \cap O_{k}\right)+\mu_{j}\left(\left\{\phi_{j}>(1+\varepsilon) a_{k}\right\} \cap O_{k}\right)<c(p, \varepsilon) \delta \mu\left(X_{k}\right) .
$$

Note that $a_{k-1} \geq a$ so $(1-\varepsilon) a_{k-1} \geq(1-\varepsilon) a>a-\varepsilon$ because $0<a<1$. By the same token $a_{k} \leq b$ so $(1+\varepsilon) a_{k} \leq(1+\varepsilon) b<b+\varepsilon$ because $0<b<1$. Hence, if 
$\phi_{j}(x)<a-\varepsilon$ then $\phi_{j}(x)<(1-\varepsilon) a_{k-1}$ and if $\phi_{j}(x)>b+\varepsilon$ then $\phi_{j}(x)>(1+\varepsilon) a_{k}$. Therefore,

$$
\mu_{j}\left(\left\{\phi_{j}<a-\varepsilon\right\} \cap O_{k}\right)+\mu_{j}\left(\left\{\phi_{j}>b+\varepsilon\right\} \cap O_{k}\right)<c(p, \varepsilon) \delta \mu\left(X_{k}\right) .
$$

If $O=\cup O_{k}$ then

$$
\mu_{j}\left(\left\{\phi_{j}<a-\varepsilon\right\} \cap O\right)+\mu_{j}\left(\left\{\phi_{j}>b+\varepsilon\right\} \cap O\right)<c(p, \varepsilon) \delta \mu(X)+\delta .
$$

Fixing $\varepsilon$ and then for any $\delta^{\prime}>0$ picking up $\delta$ so that $0<\delta<\varepsilon / 4$ and $c(p, \varepsilon) \delta \mu(X)+$ $\delta<\delta^{\prime}$ we will get a set $O$ satisfying (3).

If there are no an upper bound on $b$, then we take a constant $\beta>b$ and consider the sequence $\psi=\left\{\phi_{j} / \beta\right\}$. Evidently, $\psi_{*}=\phi_{*} / \beta$ and the measures $\left\{\left|\psi_{j}\right|^{p} \mu_{j}\right\}$ converge weak- $*$ to $\nu_{p}=\beta^{-p} \nu$. Hence $\nu_{p}=\psi_{*}^{p} \mu$.

Fix $\varepsilon, \delta>0$ and take $\varepsilon^{\prime}<\varepsilon / \beta$. By the previous result there is an open set $O$ on $K$ containing $X=\left\{a / \beta \leq \psi_{*}<b / \beta\right\}$ such that

$$
\mu_{j}\left(\left\{\psi_{j}<a / \beta-\varepsilon^{\prime}\right\} \cap O\right)+\mu_{j}\left(\left\{\psi_{j}>b / \beta+\varepsilon^{\prime}\right\} \cap O\right)<\delta
$$

or

$$
\mu_{j}\left(\left\{\phi_{j}<a-\varepsilon^{\prime} \beta\right\} \cap O\right)+\mu_{j}\left(\left\{\phi_{j}>b+\varepsilon^{\prime} \beta\right\} \cap O\right)<\delta .
$$

But $\varepsilon^{\prime} \beta<\varepsilon$ and we see that

$$
\mu_{j}\left(\left\{\phi_{j}<a-\varepsilon\right\} \cap O\right)+\mu_{j}\left(\left\{\phi_{j}>b+\varepsilon\right\} \cap O\right)<\delta .
$$

If $a=0$ then we take $0<\beta<b$ such that

$$
\frac{2 \beta \mu(G(a, b))}{(\beta+\varepsilon)(1-\beta)}<\delta
$$

If $Y=G(0, \beta)$ and $\mu(Y)>0$, then we take a closed set $C \subset Y$ such that the set $Y \backslash C$ has so small measure that there is an open set $O^{\prime}$ containing $Y \backslash C$ and of measure less than $\delta$. Then we take $f \in C(K)$ equal to 1 on $C$ and taking values between 0 and 1 elsewhere and such that

$$
\int_{K} f \phi_{*} d \mu<2 \beta \mu(Y)
$$

Let $O^{\prime \prime}=\{f>1-\beta\}$. For large $j$ we have

$$
2 \beta \mu(Y)>\int_{K} f \phi_{j} d \mu_{j} \geq(\beta+\varepsilon)(1-\beta) \mu_{j}\left(\left\{\phi_{j} \geq \beta+\varepsilon\right\} \cap O^{\prime \prime}\right) .
$$

Thus

$$
\mu_{j}\left(\{\phi \geq \beta+\varepsilon\} \cap O^{\prime \prime}\right) \leq \frac{2 \beta \mu(Y)}{(\beta+\varepsilon)(1-\beta)}<\delta .
$$

Taking $O=O^{\prime} \cup O^{\prime \prime}$ we see that it contains $Y$ and has all required properties. The set $X \backslash Y \subset G(\beta, b)$ can be handled by the previous step. 


\section{Sequences SAtisfying INTEGRAL INEQUALities}

It became clear from the previous sections that to have strong limit values the weak limit values need to exercise some control over the values of functions. The typical form of this control is

$$
\phi(z) \leq \int_{K_{0}} P(z, \zeta) \phi_{*}(\zeta) d \mu(\zeta)
$$

where $P$ is some kernel.

So let $M=\left\{\mu_{j}\right\}$ be a sequence of measures on $K$ converging weak-* to a finite measure $\mu$ on $K$ such that $\operatorname{supp} \mu_{j} \cap \operatorname{supp} \mu_{0}=\emptyset$. Let $P(z, \zeta)$ be a non-negative Borel function on $K \times K_{0}$. We require that for all $j$ and for each fixed $z \in K_{j}$ the function $P(z, \zeta)$ is bounded on $K_{0}$. Let $\mathcal{A}^{p}(M, P)$ be the set of sequences of Borel functions $\phi_{j}$ defined on $K_{j}$ which have weak limit values $\phi_{*},\|\phi\|_{L^{p}(M)}<\infty$ and (5) holds for all $z \in \cup_{j=1}^{\infty} K_{j}$.

It is reasonable to request that the class $\mathcal{A}^{p}(M, P)$ contains the constants and this is equivalent to request that

$$
\int_{K_{0}} P(z, \zeta) d \mu(\zeta)=1
$$

for all $z \in \cup_{j=1}^{\infty} K_{j}$.

We will need the following lemma.

Lemma 4.1. Let $\mu$ be a regular Borel measure on a compact space $X$. If a function $f \in L^{1}(X, \mu)$, the Borel functions $p_{j}$ on $X$ are uniformly bounded and converge weak-* to 1 , then

$$
\lim _{j \rightarrow \infty} \int_{X} f p_{j} d \mu=\int_{X} f d \mu
$$

Proof. By Lusin's theorem for every $\varepsilon>0$ we can find a closed set $Y \subset X$ such that $f$ is continuous on $Y$ and

$$
\int_{X \backslash Y}|f| d \mu<\varepsilon .
$$

Let $B$ be the uniform norm of $f$ on $Y$. We take an open set $O \subset X$ such that $Y \subset O$ and $\mu(O \backslash Y)<\varepsilon / B$. Then we extend $f$ from $Y$ to $X$ as a continuous function $h$ such that $\|h\|_{X}=B$ and $h=0$ on $X \backslash O$. Note that

$$
\int_{X}|f-h| d \mu<3 \varepsilon .
$$

Suppose that $\left|p_{j}\right| \leq A<\infty$. Then

$$
\left|\int_{X}(f-h) p_{j} d \mu\right| \leq \int_{X}\left|(f-h) p_{j}\right| d \mu \leq 3 A \varepsilon .
$$

Thus

$$
\int_{X} h p_{j} d \mu-3 A \varepsilon \leq \int_{X} f p_{j} d \mu \leq \int_{X} h p_{j} d \mu+3 A \varepsilon .
$$


Letting $j$ go to $\infty$ we get

$$
\int_{X} h d \mu-3 A \varepsilon \leq \liminf _{j \rightarrow \infty} \int_{X} f p_{j} d \mu \leq \limsup _{j \rightarrow \infty} \int_{X} f p_{j} d \mu \leq \int_{X} h d \mu+3 A \varepsilon .
$$

Thus

$$
\lim _{j \rightarrow \infty} \int_{X} f p_{j} d \mu=\int_{X} f d \mu
$$

In the following theorem we introduce an important condition on the kernel $p$ which plays a big role in the theory. It helps to prove a theorem establishing the existence of strong boundary values for sequences in $\mathcal{A}^{p}(M, P)$ and provides important estimates. For the future, if $h$ is a function on $K_{0}$ we set the sequence $P h$ consisting of functions

$$
(P h)_{j}(z)=\int_{K} P(z, \zeta) h(\zeta) d \mu(\zeta), \quad z \in K_{j}
$$

Theorem 4.2. Suppose that the kernel $P(z, \zeta)$ satisfies (6), the functions

$$
p_{j}(\zeta)=\int_{K_{j}} P(z, \zeta) d \mu_{j}(z)
$$

are uniformly bounded and converge weak-* to 1 on $K_{0}$. Then:

(1) any sequence $\left\{\phi_{j}\right\} \in \mathcal{A}^{p}(M, P), p>1$, has strong limit values equal to $\phi_{*}$ and $\|\phi\|_{L^{p}(M)}=\left\|\phi_{*}\right\|_{L^{p}(K)}$;

(2) if $h \in L^{p}\left(K_{0}, \mu\right)$ then $\|P h\|_{L^{p}(M)} \leq\|h\|_{L^{p}\left(K_{0}\right)}$;

(3) if the sequence $P h$ has strong limit values equal to $h$ for a dense set of functions $h$ in $L^{p}\left(K_{0}, \mu\right)$ then the same holds for any function in $L^{p}\left(K_{0}, \mu\right)$, $p>1$.

Proof. 1) Let $\phi \in \mathcal{A}^{p}(M, P)$. For $z \in K_{j}$ we define

$$
\psi_{j}(z)=\int_{K_{0}} P(z, \zeta) \phi_{*}(\zeta) d \mu(\zeta)
$$

By $(5) \psi_{j} \geq \phi_{j}$ for all $j$. Moreover,

$$
\int_{K_{j}} \psi_{j}(z) d \mu_{j}(z)=\int_{K_{0}} \phi_{*}(\zeta)\left(\int_{K_{j}} P(z, \zeta) d \mu_{j}(z)\right) d \mu(\zeta)=\int_{K_{0}} \phi_{*}(\zeta) p_{j}(\zeta) d \mu(\zeta)
$$

and

$$
\int_{K_{j}}\left|\psi_{j}(z)\right| d \mu_{j}(z) \leq \int_{K_{0}}\left|\phi_{*}(\zeta)\right|\left(\int_{K_{j}} P(z, \zeta) d \mu_{j}(z)\right) d \mu(\zeta)=\int_{K_{0}}\left|\phi_{*}(\zeta)\right| p_{j}(\zeta) d \mu(\zeta) .
$$

Hence the norms $\left\|\psi_{j}\right\|_{L^{1}\left(K_{j}\right)}$ are uniformly bounded and we can take a subsequence $\left\{\psi_{j_{k}} \mu_{j_{k}}\right\}$ converging weak-* to a measure $\nu$. Then $\nu \geq \phi_{*} \mu$ but by Lemma 4.1

$$
\int_{K_{0}} d \nu=\lim _{k \rightarrow \infty} \int_{K_{j_{k}}} \psi_{j_{k}} d \mu_{j_{k}}=\lim _{k \rightarrow \infty} \int_{K_{0}} \phi_{*} p_{j_{k}} d \mu=\int_{K_{0}} \phi_{*} d \mu .
$$


Thus $\nu=\phi_{*} \mu$ and the sequence $\left\{\psi_{j} \mu_{j}\right\}$ converges weak-* to $\phi_{*} \mu$.

Therefore, the non-negative sequence $\left\{\psi_{j}-\phi_{j}\right\}$ has the zero weak- $*$ limit values. By Chebyshev's inequality the sequence $\left\{\psi_{j}-\phi_{j}\right\}$ has the strong boundary values equal to 0 .

Now by the definition of $\psi$, Hölder's inequality and (6)

$$
\int_{K_{j}}\left|\psi_{j}\right|^{p} d \mu_{j}=\int_{K_{j}}\left|\int_{K_{0}} P(z, \zeta) \phi_{*}(\zeta) d \mu(\zeta)\right|^{p} d \mu_{j}(z) \leq \int_{K_{0}}\left|\phi_{*}\right|^{p} p_{j} d \mu .
$$

Thus $\|\phi\|_{L^{p}(M)} \leq\left\|\phi_{*}\right\|_{L^{p}(K)}$. But by Lemma $2.1\|\phi\|_{L^{p}(M)} \geq\left\|\phi_{*}\right\|_{L^{p}(K)}$. Hence $\|\phi\|_{L^{p}(M)}=\left\|\phi_{*}\right\|_{L^{p}(K)}$.

As before we derive that the sequence $\left\{\left|\psi_{j}\right|^{p} \mu_{j}\right\}$ is bounded and if a subsequence $\left\{\left|\psi_{j_{k}}\right|^{p} \mu_{j_{k}}\right\}$ converges weak-* to a measure $\nu_{p}$, then

$$
\int_{K} d \nu_{p} \leq \int_{K}\left|\phi_{*}\right|^{p} d \mu
$$

By Theorem $2.2 \nu_{p}=\left|\phi_{*}\right|^{p} \mu$ and this implies that the sequence $\left\{\left|\psi_{j}\right|^{p} \mu_{j_{k}}\right\}$ converges weak-* to a measure $\nu_{p}$. By Theorem 3.6 the sequence $\left\{\psi_{j}\right\}$ has strong boundary values equal to $\phi_{*}$. Since $\phi=(\phi-\psi)+\psi$ by Theorem 3.3(2) $\phi$ has the strong limit values equal to $\phi_{*}$.

2)

$$
\begin{aligned}
& \int_{K_{j}}\left|(P h)_{j}(z)\right|^{p} d \mu_{j}(z)=\iint_{K_{j}}\left|\int_{K_{0}} h(\zeta) P(z, \zeta) d \mu(\zeta)\right|^{p} d \mu_{j}(z) \\
& \leq \int_{K_{j}}\left(\int_{K_{0}}|h(\zeta)|^{p} P(z, \zeta) d \mu(\zeta)\left(\int_{K_{0}} P(z, \zeta) d \mu(\zeta)\right)^{p / q}\right) d \mu_{j}(z) \\
& =\int_{K_{j}}\left(\int_{K_{0}}|h(\zeta)|^{p} P(z, \zeta) d \mu(\zeta)\right) d \mu_{j}(z) \\
& =\int_{K_{0}}|h(\zeta)|^{p}\left(\int_{K_{j}} P(z, \zeta) d \mu(z)\right) d \mu(\zeta)=\int_{K_{0}}|h(\zeta)|^{p} p_{j}(\zeta) d \mu(\zeta) .
\end{aligned}
$$

By Lemma 4.1 the last integrals converge to $\|h\|_{L^{p}\left(K_{0}\right)}^{p}$.

3) If $\phi \in L^{p}\left(K_{0}, \mu\right)$ then for every $\varepsilon>0$ there is function $h \in L^{p}\left(K_{0}, \mu\right)$ such that $P h$ has strong limit values $h$ and $\|h-\phi\|_{L^{p}\left(K_{0}, \mu\right)}<\varepsilon$. By 2) $\|P(h-\phi)\|_{L^{p}(M)} \leq \varepsilon$. By the last statement in Theorem 3.4 the sequence $P \phi$ has strong limit values equal to $\phi$.

So we see the importance of the condition on the functions $p_{j}$ imposed in Theorem 4.2. Let us list two important cases when these conditions hold at least partially.

Theorem 4.3. Suppose that the kernel $P(z, \zeta)$ satisfies (6).

1)If the measures $P\left(z_{j}, \zeta\right) \mu(\zeta)$ converge weak-* to $\delta_{\zeta_{0}}$ when the sequence $\left\{z_{j}\right\} \subset$ $K$ converges to $\zeta_{0} \in K_{0}$, then the functions $p_{j}(\zeta)$ converge weak-* to 1 on $K_{0}$. Moreover, if $h \in C\left(K_{0}\right)$ then the functions $(P h)_{j}$ converge uniformly to $h$. 
2) If the measures $P\left(z, \zeta_{j}\right) \mu_{j}(z)$ converge weak-* to $\delta_{\zeta}$ when the sequence $\left\{\zeta_{j}\right\} \subset$ $K_{0}$ converges to $\zeta \in K_{0}$, then the functions $p_{j}(\zeta)$ are uniformly bounded and converge weak-* to 1 on $K_{0}$. Moreover, if $h \in L^{p}(\mu), p>1$, then the sequence Ph belongs to $\mathcal{A}^{p}(M, P)$ and has weak (and, consequently, strong) limit values equal to $h$.

Proof. 1) Let $h \in C\left(K_{0}\right)$. Let us show that for every $\varepsilon>0$ there is $j_{0}$ and $\delta>0$ such that $\left|(P h)_{j}(z)-h(\zeta)\right|<\varepsilon$ when $j \geq j_{0}$ and $|z-\zeta|<\delta$. Due to the compactness of $K$ the negation of this statement means that there is $\zeta_{0} \in K_{0}$ and a sequence $z_{j_{k}} \in K_{j_{k}}$ such that $\left|z_{j_{k}}-\zeta_{0}\right| \rightarrow 0$ as $k \rightarrow \infty$ but $\left|(P h)_{j}\left(z_{j_{k}}\right)-h\left(\zeta_{0}\right)\right|>\varepsilon>0$. But it clearly contradicts to the imposed condition on the measures $P\left(z_{j}, \zeta\right) \mu(\zeta)$.

Hence, if $H$ is a continuous extension of $h$ to $K$ we may assume that $\mid(P h)_{j}(z)-$ $H(z) \mid<\varepsilon$ when $j \geq j_{0}$. Therefore,

$$
\int_{K_{0}} h(z) d \mu(\zeta)=\lim _{j \rightarrow \infty} \int_{K_{j}} H(z) d \mu_{j}(z)=\lim _{j \rightarrow \infty} \int_{K_{j}}(P h)_{j}(z) d \mu_{j}(z) .
$$

But

$$
\int_{K_{j}}(P h)_{j}(z) d \mu_{j}(z)=\int_{K_{0}} h(\zeta)\left(\int_{K_{j}} P(z, \zeta) d \mu_{j}(z)\right) d \mu(\zeta)=\int_{K_{0}} h(\zeta) p_{j}(\zeta) d \mu(\zeta) .
$$

Hence,

$$
\lim _{j \rightarrow \infty} \int_{K_{0}} h(\zeta) p_{j}(\zeta) d \mu(\zeta)=\int_{K_{0}} h(z) d \mu(\zeta)
$$

and we are done.

2) Clearly, the functions $p_{j}(\zeta)$ converge to 1 pointwise on $K_{0}$. To show that they are uniformly bounded we suppose that there is a sequence $\left\{j_{k}\right\}$ and points $\zeta_{k} \in \operatorname{supp} \mu$ such that $p_{j_{k}}\left(\zeta_{k}\right) \rightarrow \infty$ as $k \rightarrow \infty$. Without any loss of generality we may assume that points $\zeta_{k}$ converge to $\zeta_{0}$. Since the measures $P\left(z, \zeta_{k}\right) \mu_{j}(z)$ converge weak-* to $\delta_{\zeta_{0}}$ we got a contradiction.

Now if $f \in C(K)$ then

$$
\int_{K_{j}} f(z)(P h)_{j}(z) d \mu_{j}(z)=\int_{K} h(\zeta)\left(\int_{K_{j}} f(z) P(z, \zeta) d \mu_{j}(z)\right) d \mu(\zeta) .
$$

The functions

$$
F_{j}(\zeta)=\int_{K_{j}} f(z) P(z, \zeta) d \mu_{j}(z)
$$

are uniformly bounded and converge to $f(\zeta)$ pointwise. Hence

$$
\lim _{j \rightarrow \infty} \int_{K_{j}} f(z)(P h)_{j}(z) d \mu_{j}(z)=\int_{K} h(\zeta) f(\zeta) d \mu(\zeta) .
$$

Thus the weak limit values of $\phi$ is $h$.

Now

$$
\int_{K_{j}}\left|(P h)_{j}\right|^{p} d \mu_{j} \leq \int_{K}|h|^{p} p_{j} d \mu
$$


and we see that $\|P h\|_{L^{p}(M)} \leq\|h\|_{L^{p}(\mu)}$. Thus $P h \in \mathcal{A}^{p}(M, P)$ and has the strong limit values equal to $h$.

Generally, the inequality (5) comes from similar inequalities obtained on the interior of a domain. This process is described as follows:

Theorem 4.4. Suppose that for each $j$ there are Borel functions $P_{j}(z, \zeta)$ defined on $\cup_{m=1}^{j-1} K_{m} \times K_{j}$ such that for each $z \in K_{m}$ the functions $P_{j}(z, \cdot), j>m$, are uniformly bounded and have the strong limit values $P(z, \cdot)$ with respect to $M=\left\{\mu_{j}\right\}$.

If $\phi \in \mathcal{A}^{p}(M), p>1$, and

$$
\phi_{m}(z) \leq \int_{K_{j}} P_{j}(z, \zeta) \phi_{j}(\zeta) d \mu_{j}(\zeta)
$$

for each $z \in \cup_{m=1}^{j-1} K_{m}$, then $\phi \in \mathcal{A}^{p}(M, P)$.

Proof. Let us fix some $z \in K_{m}$ and let $q_{j}(\zeta)=P_{j}(z, \zeta)$. The sequence $\left\{q_{j}\right\} \in$ $L^{\infty}(M)$ and the sequence $\phi \in L^{p}(M), p>1$. By Theorem 3.5(2) the sequence $\left\{\phi_{j} q_{j} \mu_{j}\right\}$ converges weak- $*$ to $\phi_{*} P(z, \cdot) \mu$. Hence

$$
\phi(z) \leq \int_{K_{0}} P(z, \zeta) \phi_{*}(\zeta) d \mu(\zeta)
$$

\section{BOUNDARY VALUES}

These results can be applied to the theory of boundary values in the following manner. Suppose that $D$ is a bounded domain in $\mathbb{R}^{n}$ exhausted by domains $D_{r}$, $r<0$, such that $D_{r} \subset \subset D_{s}$ when $s>r$ and $D=D_{0}=\cup_{r<0} D_{r}$. Suppose also that there are measures $\mu_{r}$ supported by $S_{r}=\partial D_{r}$ converging weak-* in $C^{*}(\bar{D})$ as $r \rightarrow 0^{-}$to a finite measure $\mu$ supported by $\partial D$. Let $K_{0}=\operatorname{supp} \mu$.

We say that a function $u$ on $D$ has boundary values with respect to measures $\mu_{r}$ if it has strong limit values with respect to $M=\left\{\mu_{r_{j}}\right\}$ for any sequence $r_{j} \nearrow 0$ and these strong limit values do not depend on the choice of a sequence.

For $p \geq 0$ and a continuous function $u$ on $D$ we define

$$
\|u\|_{p}=\limsup _{r \rightarrow 0^{-}} \int_{S_{r}}|u|^{p} d \mu_{r} .
$$

We assume that a Borel function $P(z, \zeta)$ is defined on $D \times \partial D$. We will require the kernel $P(z, \zeta)$ to satisfy the following conditions:

(P1)

$$
\int_{\partial D} P(z, \zeta) d \mu(\zeta)=1
$$

(P2) for some $p>0$ and every $h \in L^{p}\left(K_{0}, \mu\right)$ the function

$$
P h(z)=\int_{\partial D} h(z) P(z, \zeta) d \mu(\zeta)
$$

has boundary values equal to $h$ and $\|P h\|_{p} \leq\|h\|_{L^{p}\left(K_{0}, \mu\right)}$.

To check the most complicated condition (P2) we note that by Theorem 4.2 it holds if we assume that: 
(1) for every sequence $r_{j} \nearrow 0$ the functions

$$
p_{r_{j}}(\zeta)=\int_{S_{r_{j}}} P(z, \zeta) d \mu_{r_{j}}(z)
$$

are uniformly bounded and converge weak-* to 1 on $K_{0}$;

(2) the set of $h \in L^{p}\left(K_{0}, \mu\right)$ such that the function $P h$ has strong boundary values equal to $h$ with respect to any sequence $M=\left\{\mu_{r_{j}}\right\}$ is dense in $L^{p}\left(K_{0}, \mu\right)$.

The list of cases when (1) holds is given in Theorem 4.3. The second condition will follow if, for example, it holds for functions in $C\left(K_{0}\right)$.

Theorem 5.1. Let $D$ be a domain in $\mathbb{R}^{n}$. Let $\mathcal{F}^{p}, p>1$, be a set of continuous functions $u$ on $D$ and let $P$ be a Borel function on $D \times \partial D$ such that:

(1) the kernel $P$ satisfies conditions (P1)-(P2);

(2) $\|u\|_{p}<\infty$ for all $u \in \mathcal{F}^{p}$;

(3) if $u \in \mathcal{F}^{p}$ and the functions $\left.u\right|_{S_{r_{j}}}$ have weak limit values $\psi$ for some sequence $r_{j} \nearrow 0$, then $u \leq P \psi$ on $D$.

If $u \in \mathcal{F}^{p}$ then it has boundary values.

Proof. Suppose that the functions $u_{r_{j}}$ have weak limit values $\psi$ with respect to $M=\left\{\mu_{r_{j}}\right\}$ for some sequence $r_{j} \nearrow 0$. By Lemma $2.1 \psi \in L^{p}\left(K_{0}, \mu\right)$ and by (3) $u \leq P \psi$ on $D$. Hence the function $v=u-P \psi$ is non-positive and by (P2) has weak boundary values with respect to $M$ equal to 0 . Thus $v$ has strong limit values with respect to $M$ equal to 0 and we see that $u$ has strong limit values with respect to $M=\left\{\mu_{r_{j}}\right\}$ equal to $\psi$.

Now suppose that $u$ has strong limit values $\phi$ with respect to $N=\left\{\mu_{t_{j}}\right\}, t_{j} \nearrow$ 0 . The functions $v_{j}=\left.v\right|_{S_{t_{j}}}$ are non-positive and $\|v\|_{p}<\infty$. Hence there is a subsequence $N^{\prime}=\left\{\mu_{t_{j_{k}}}\right\}$ such that $v$ has weak limit values $v_{*}$ with respect to $N^{\prime}$. Clearly, $v_{*} \leq 0$. Thus by (P2)

$$
\phi=\left\{\left.u\right|_{S_{t_{j_{k}}}}\right\}_{*}=\left\{\left.(P \psi+v)\right|_{S_{t_{j_{k}}}}\right\}_{*}=\psi+v_{*}
$$

and we see that $\phi \leq \psi$. By the symmetry $\psi \leq \phi$ and we see that $\phi=\psi$.

If $u \in \mathcal{F}^{p}$ and $r_{j} \rightarrow 0^{-}$then by the first part of the proof all subsequences of $\left\{u_{r_{j}}\right\}$ which have weak limit values have strong limit values. By the second part of the proof these values don't depend on the subsequence. Hence the sequence $\left\{u_{r_{j}}\right\}$ has weak limit values and, consequently, strong limit values which do not depend on the sequence. Hence, $u$ has boundary values.

In this theorem the third condition is the most difficult to verify (provided (P2) is checked). The only strategy for verification we know is the following. Suppose that for each $r$ the kernels $P_{r}(z, \zeta)$ are defined on $D_{r} \times S_{r}$ and are Borel functions. We will require the kernels $P_{r}(z, \zeta)$ to satisfy the following conditions:

(1) for each $z \in D$ the function $P_{r}(z, \zeta)$ is non-negative and uniformly bounded on $S_{r}$ when $r$ is close to 0 ;

(2) $P_{r} u \geq u$ on $D_{r}$ for every $u \in \mathcal{F}^{p}$;

(3) for each $z \in D$ the kernel $P(z, \cdot)$ is the strong limit values of the functions $P_{r_{j}}(z, \cdot)$ with respect to any sequence $M=\left\{\mu_{r_{j}}\right\}, r_{j} \nearrow 0$. 
Theorem 5.2. If the conditions above hold, $u \in \mathcal{F}^{p}$ and the functions $\left.u\right|_{S_{r_{j}}}$ have weak limit values $u_{*}$ for some sequence $r_{j} \nearrow 0$, then $u \leq P u_{*}$ on $D$ and, consequently, $u$ has boundary values.

Proof. The proof repeats the proof of Theorem 4.4. Let us fix some $z \in D$ and let $q_{j}(\zeta)=P_{r_{j}}(z, \zeta)$. Consider $M=\left\{\mu_{r_{j}}\right\}$. Then the sequence $\left\{u_{j}=\left.u\right|_{S_{r_{j}}}\right\}$ belongs to $L^{p}(M), p>1$, and the sequence $\left\{q_{j}\right\} \in L^{\infty}(M)$. By Theorem 3.5(2) the sequence $\left\{u_{j} q_{j} \mu_{r_{j}}\right\}$ converges weak-* to $u_{*} P(z, \cdot) \mu$. Hence

$$
u(z) \leq \int_{\partial D} P(z, \zeta) u_{*}(\zeta) d \mu(\zeta)
$$

To check the third condition in the list above the following result can be helpful. Assuming that $P=P_{0}, \mu=\mu_{0}$ and $\partial D=S_{0}$ we introduce the function

$$
Q_{r}(z, w)=\int_{S_{r}} P_{r}(z, \zeta) P_{r}(w, \zeta) d \mu_{r}(\zeta) .
$$

Theorem 5.3. Suppose that:

(1) for each $z \in D$ the functions $P_{r}(z, \zeta)$ are non-negative and uniformly bounded on $S_{r}$ when $r$ is close to 0 ;

(2) for each $z \in D$ the kernel $P(z, \cdot)$ is the weak limit values of the functions $P_{r_{j}}(z, \cdot)$ with respect to any sequence $M=\left\{\mu_{r_{j}}\right\}, r_{j} \nearrow 0$;

(3) $Q_{0}(z, z)=\lim _{r \rightarrow 0^{-}} Q_{r}(z, z)$ for every $z \in D$.

Then for each $z \in D$ the kernel $P(z, \cdot)$ is the strong limit values of the functions $P_{r_{j}}(z, \cdot)$ with respect to any sequence $M=\left\{\mu_{r_{j}}\right\}, r_{j} \nearrow 0$.

Proof. Suppose that the sequence $\left\{P_{r_{j}}^{2}(z, \cdot)\right\}$ has weak limit values equal to $\phi$ with respect to a sequence $M=\left\{\mu_{r_{j}}\right\}$. Then

$$
\int_{K_{0}} \phi d \mu=\lim _{j \rightarrow \infty} \int_{S_{r}} P_{r_{j}}^{2}(z, \zeta) d \mu_{r_{j}}(\zeta)=\lim _{j \rightarrow \infty} Q_{r_{j}}(z, z)=Q_{0}(z, z)=\int_{K_{0}} P_{0}^{2}(z, \zeta) d \mu(\zeta) .
$$

By Theorem 3.6 $P(z, \cdot)$ is the strong limit values of the functions $P_{r_{j}}(z, \cdot)$ with respect to $M$.

Since the measures $P_{r}^{2}(z, \cdot) \mu_{r}$ are uniformly bounded any sequence $\left\{\mu_{r_{j}}\right\}, r_{j} \nearrow 0$, has a weak-* converging subsequence. But weak- $*$ limits of these sequences coincide and, therefore, for each $z \in D$ the kernel $P(z, \cdot)$ is the strong limit values of the functions $P_{r_{j}}(z, \cdot)$ with respect to any sequence $M=\left\{\mu_{r_{j}}\right\}, r_{j} \nearrow 0$.

As the following theorem shows the existence of boundary values allows us to expand the integral representation formulas from subdomains to the whole domain. The proof follows immediately from the second part of Theorem 3.5.

Theorem 5.4. Suppose that for each $z \in D$ the functions $P_{r}(z, \zeta)$ are non-negative and uniformly bounded on $S_{r}$ when $r$ is close to 0 and the kernel $P(z, \cdot)$ is the weak limit values of the functions $P_{r_{j}}(z, \cdot)$ with respect to some sequence $M=\left\{\mu_{r_{j}}\right\}$, $r_{j} \nearrow 0$. Suppose also that $\mathcal{H}$ is a set of continuous functions $u$ on $D$ such that for any $u \in \mathcal{H}$ : 
(1)

$$
u(z)=\int_{S_{r}} u(\zeta) P_{r}(z, \zeta) d \mu_{r}(\zeta)+L_{r}(z, u),
$$

for all $z \in D_{r}$ and $r<0$;

(2) $\|u\|_{p}<\infty$ for some $p>1$;

(3) there are boundary values $u^{*}$.

If $L_{r}(z, u) \leq 0$ and $\lim _{r \rightarrow 0^{-}} L_{r}(z, u)=L(z, u)$ exists for all $z \in D$, then

$$
u(z)=\int_{S_{0}} u^{*}(\zeta) P(z, \zeta) d \mu(\zeta)+L(z, u) .
$$

In the last statement we show that when functions from some class have radial limits then they have boundary values. By radial limits we mean the following: a continuous mapping $v$ of $K_{0} \times[0, \varepsilon), \varepsilon>0$, into $\mathbb{R}^{n}$ is called radial if:

(1) $v(\zeta, 0)=\zeta$;

(2) there is $r_{0}<0$ such that for every $\zeta \in K_{0}$ the set $\left\{t: v(\zeta, t) \in S_{r}\right\} \neq \emptyset$ when $r_{0}>r>0$, and the functions $t_{r}(\zeta)=\inf \left\{t: v(\zeta, t) \in S_{r}\right\}$ are continuous and converging uniformly to 0 as $r \nearrow 0$;

(3) there is a constant $c>0$ such that if a Borel set $E \subset K_{0}$ and $E_{r}=\{z \in$ $\left.S_{r}: z=v\left(\zeta, t_{r}(\zeta)\right), \zeta \in E\right\}$, then $\lim \sup _{r \rightarrow 0^{-}} \mu_{r}\left(E_{r}\right) \leq c \mu(E)$

(4) if $A_{r}=\left\{z \in S_{r}: z=v\left(\zeta, t_{r}(\zeta)\right), \zeta \in K_{0}\right\}$ then $\limsup _{r \rightarrow 0^{-}} \mu_{r}\left(S_{r} \backslash A_{r}\right)=0$.

We say that a function $u$ on $D$ has radial limits $\widetilde{u} \mu$-a.e. with respect to a radial mapping $v$ if there is a function $\widetilde{u}$ on $K_{0}$ such that $\lim _{t \rightarrow 0} u\left(v\left(\zeta, t_{r}(\zeta)\right)\right)$ exists $\mu$-a.e and is equal to $\widetilde{u}(\zeta) \mu$-a.e.

Theorem 5.5. If a Borel function $u$ on $D$ has radial limits $\mu$-a.e. with respect to a radial mapping $v$, then $u$ has boundary values equal to $\widetilde{u}$.

Proof. First of all, we note that by Lemma 2.4 for any sequence $r_{j} \nearrow 0$ the measures $\mu_{r_{j}}$ converge to $\mu$ regularly. So we can use the last part of Theorem 3.4. Given a function $u$ on $D$ with radial limits $\widetilde{u}$ we fix $\varepsilon, \delta>0$ and take a continuous function $f$ on $K_{0}$ such that $f=\widetilde{u}$ on a set $E$ with $\mu(E)>\mu\left(K_{0}\right)-\delta$. Let us denote by the same letter $f$ the continuous extension of $f$ to $D$.

Since the functions $t_{r}$ converge uniformly to 0 there are $r_{0}<0$ and a set $F \subset K_{0}$ such that $\mu(F)>\mu\left(K_{0}\right)-\delta$,

$$
\sup _{0<r<r_{0}}\left|\widetilde{u}(\zeta)-u\left(v\left(\zeta, t_{r}(\zeta)\right)\right)\right|<\varepsilon
$$

and

$$
\left.\sup _{0<r<r_{0}} \mid \tilde{f} \zeta\right)-f\left(v\left(\zeta, t_{r}(\zeta)\right)\right) \mid<\varepsilon
$$

for all $\zeta \in F$.

Let $G_{r}$ be the set of points $z$ in $S_{r}$ such that $z=v\left(\zeta, t_{r}(\zeta)\right)$ for some $\zeta \in F \cap E$. By the property (3) of radial mappings we can find negative $r_{1} \geq r_{0}$ such that $\mu_{r}\left(S_{r} \backslash A_{r}\right)<\delta$ when $r>r_{1}$. By the property (2) of radial mappings we can find negative $r_{2} \geq r_{1}$ such that $\left.\mu\left(K_{0} \backslash(E \cap F)\right)_{r}\right)<2 c \delta$ when $r>r_{2}$. Hence

$$
\mu_{r}(\{|u(\zeta)-f(\zeta)|>2 \varepsilon\}) \leq(2 c+1) \delta .
$$

Hence $u$ has boundary values equal to $\widetilde{u}$. 


\section{BOUNDARY VALUES OF HARMONIC FUNCTIONS}

The simplest case is the case of harmonic functions on regular domains, i.e., bounded domains such that any continuous function $\phi$ on their boundaries has a harmonic extension $h_{\phi}$ to the domain continuous up to the boundary and this extension coincides with $\phi$ on the boundary.

Let $D$ be such a domain. For any $x \in D$ the evaluation $h_{\phi}(x)$ defined for $\phi \in C(\partial D)$ is a continuous linear functional by the maximum principle and, consequently, there are harmonic measures $\mu_{x}$ on $\partial D$ such that

$$
h_{\phi}(x)=\int_{\partial D} \phi d \mu_{x}
$$

For any $\zeta \in \partial D$ let $S_{\zeta, r}$ be the intersection of $\partial D$ with the closed ball centered at $\zeta$ and of radius $r>0$ and let $\chi_{\zeta, r}$ be the characteristic function of $S_{\zeta, r}$. Since $\chi_{\zeta, r}$ is the limit of a decreasing sequence of functions in $C(\partial D)$, the function $F_{r}(x, \zeta)=$ $\mu_{x}\left(S_{\zeta, r}\right)$ is harmonic on $D$ and, clearly, for any $\zeta \in \partial D$ extends continuously as 0 on $\partial D \backslash S_{\zeta, r}$ and as 1 on the relative interior of $S_{\zeta, r}$ in $\partial D$. We define $F_{r}(x, \zeta)$ on $\bar{D} \times \partial D$ letting it to be equal to 1 when $x \in S_{\zeta, r}$. So $F_{r}$ is a Borel function on $\bar{D} \times \partial D$

Let us fix $x_{0} \in D$ and define

$$
P(x, \zeta, r)=\frac{F_{r}(x, \zeta)}{F_{r}\left(x_{0}, \zeta\right)} .
$$

By Harnack's inequality for every compact set $K \subset D$ there is a constant $C(K)>$ 0 such that $C^{-1}(K) F_{r}(x, \zeta) \leq F_{r}\left(x_{0}, \zeta\right) \leq C(K) F_{r}(x, \zeta)$ when $x, x_{0} \in K$ and $\zeta \in \partial D$. Hence the function

$$
P(x, \zeta)=\limsup _{r \rightarrow 0^{-}} P(x, \zeta, r)
$$

is Borel on $D \times \partial D, C^{-1}(K) \leq P(x, \zeta) \leq C(K)$ when $x, x_{0} \in K$ and $\zeta \in \partial D$ and, again by Harnack's inequality, $P(x, \zeta)$ is continuous in $x$. Thus $P(x, \zeta)$ is subharmonic in $x$. Moreover, $\mu_{x}(\zeta)=P(x, \zeta) \mu_{x_{0}}(\zeta)$ for any $x \in D$. Hence

$$
h_{\phi}(x)=\int_{\partial D} \phi(\zeta) P(x, \zeta) d \mu_{x_{0}} .
$$

Let $V$ be the volume element on $D$. We cover $D$ by a countable family of closed balls $B\left(x_{j}, r_{j}\right)$ centered at $x_{j}$ and of radius $r_{j}>0$ such that $B\left(x_{j}, r_{j}\right) \subset D$. For every $j$ and $\zeta \in \partial D$ let

$$
\Psi_{j}(x, \zeta)=\frac{1}{V\left(B\left(x_{j}, r_{j}\right)\right)} \int_{B\left(x_{j}, r_{j}\right)} P(y, \zeta) d V .
$$

Then

$$
h_{\phi}\left(x_{j}\right)=\frac{1}{V\left(B\left(x_{j}, r_{j}\right)\right)} \int_{B\left(x_{j}, r_{j}\right)} h_{\phi}(y) d V=\int_{\partial D} \phi(\zeta) \Psi_{j}\left(x_{j}, \zeta\right) d \mu_{x_{0}} .
$$

Since it is true for every $\phi \in C(\partial D)$ we see that the equality $\Psi_{j}\left(x_{j}, \zeta\right)=P\left(x_{j}, \zeta\right)$ holds for all $\zeta \in \partial D$ except of a set $E_{j}$ with $\mu_{x_{0}}\left(E_{j}\right)=0$. But the subharmonicity of $P(x, \zeta)$ yields that $P(y, \zeta)$ is harmonic on $B\left(x_{j}, r_{j}\right)$ for all $\zeta \in \partial D \backslash E_{j}$. Hence 
the function $P(x, \zeta)$ is harmonic in $x$ for $\mu_{x_{0}}$-almost all $\zeta$ and is the Poisson kernel on $D$ centered at $x_{0}$.

Now suppose that $D$ is exhausted by domains $\left\{D_{r}\right\}, r<0$. Let $\mu_{r, x}$ and $P_{r}(x, \zeta)$ be the harmonic measure and the Poisson kernel centered at $x_{0}$ respectively on $D_{r}$. Note that $P\left(x_{0}, \zeta\right)=P_{r}\left(x_{0}, \zeta\right)=1$. Hence by Harnack's inequality for every compact set $F \subset D$ there is a constant $C(F)>0$ such that $C^{-1}(F) \leq P_{r}(x, \zeta) \leq$ $C(F)$ when $x \in F$.

Since for any $\phi \in C(\bar{D})$ and any $x \in D$

$$
\lim _{r \rightarrow 0^{-}} \int_{S_{r}} \phi(\zeta) P_{r}(x, \zeta) d \mu_{r, x_{0}}(\zeta)=\lim _{r \rightarrow 0^{-}} \int_{S_{r}} h_{\phi}(\zeta) P_{r}(x, \zeta) d \mu_{r, x_{0}}(\zeta)=h_{\phi}(x),
$$

we see that the measures $P_{r}(x, \zeta) d \mu_{r, x_{0}}(\zeta)$ converge weak-* to $P(x, \zeta) d \mu_{x_{0}}(\zeta)$. In particular, since $P_{r}\left(x_{0}, \zeta\right)=P\left(x_{0}, \zeta\right) \equiv 1$ the measures $\mu_{r, x_{0}}(\zeta)$ converge weak-* to $\mu_{x_{0}}(\zeta)$.

In the future $p \geq 1$ and we let $\mu_{r, x_{0}}=\mu_{r}$ and $\mu_{x_{0}}=\mu$. We define the space $\mathcal{S}^{p}(D)$ as the space of all continuous subharmonic functions $u$ on $D$ such that

$$
\|u\|_{\mathcal{S}^{p}(D)}^{p}=\limsup _{r \rightarrow 0^{-}} \int_{S_{r}}|u|^{p} d \mu_{r}<\infty .
$$

The following theorem shows that any function in $L^{p}\left(\partial D, \mu_{x_{0}}\right)$ is the trace of a function in $\mathcal{S}^{p}(D)$ and the Poisson integral serves as restoring operator.

Theorem 6.1. Let $D$ be a regular domain in $\mathbb{R}^{n}$ and $p>1$. For every function $\phi \in L^{p}\left(\partial D, \mu_{x_{0}}\right)$ there is a unique harmonic function $h_{\phi} \in \mathcal{S}^{p}(D)$ with boundary values equal to $\phi$ and $\left\|h_{\phi}\right\|_{\mathcal{S}^{p}(D)} \leq\|\phi\|_{L^{p}\left(\partial D, \mu_{x_{0}}\right)}$. Moreover, $h_{\phi}=P \phi$.

Proof. Note that

$$
\int_{\partial D} P(x, \zeta) d \mu(\zeta) \equiv 1
$$

so the condition (P1) from Section 5 holds. Since $P(x, \zeta)$ is harmonic for $\mu$-almost all $\zeta$ we see that

$$
p_{r}(\zeta)=\int_{\partial D} P_{r}(x, \zeta) d \mu_{r}(\zeta)=P_{r}\left(x_{0}, \zeta\right)=1 .
$$

Moreover, if $h \in C(\partial D)$ then the function $P h$ has boundary values equal to $h$ with respect to any sequence $M=\left\{\mu_{r_{j}}\right\}$. Since $C(\partial D)$ is dense in $L^{p}\left(\partial D, \mu_{x_{0}}\right)$, by Theorem 4.2 for every $\phi \in L^{p}\left(\partial D, \mu_{x_{0}}\right)$ the function

$$
h_{\phi}(x)=P \phi(x)=\int_{\partial D} \phi(x) P(x, \zeta) d \mu(\zeta)
$$

has boundary values equal to $h$ with respect to any sequence $M=\left\{\mu_{r_{j}}\right\}$ and $\|P \phi\|_{p} \leq\|\phi\|_{L^{p}\left(\partial D, \mu_{x_{0}}\right)}$. Thus the condition (P2) holds also.

If $u \in \mathcal{S}^{p}(D)$ is another function with boundary values $\phi$, then we take a sequence $\left\{r_{j} \nearrow 0\right\}$. The weak limit of functions $P_{r_{j}}(x, \zeta)$ with respect to $\left\{\mu_{r_{j}}\right\}$ is equal to $P(x, \zeta)$ for all $x \in D$. By Theorem 3.5(2)

$$
u(x)=\lim _{j \rightarrow \infty} \int_{\partial D_{r_{j}}} u(y) P_{r}(x, y) d \mu_{r_{j}}(y)=\int_{\partial D} \phi(\zeta) P(x, \zeta) d \mu(\zeta) .
$$


Thus $u=P \phi$.

By the Riesz Representation Theorem every subharmonic function $u$ on $D$ can be represented on $D_{r}$ as

$$
u(x)=\int_{S_{r}} u(y) P_{r}(x, y) d \mu_{r}(y)+\int_{D_{r}} G_{D_{r}}(x, y) \Delta u(y),
$$

where $G_{D_{r}}(x, y)$ is the Green kernel on $D_{r}$. For functions in $\mathcal{S}^{p}(D)$ this property is characteristic for functions with boundary values.

Theorem 6.2. A function $u \in \mathcal{S}^{p}(D)$ has the representation

$$
u(x)=\int_{S} \phi(y) P(x, y) d \mu(y)+\int_{D} G_{D}(x, y) \Delta u(y) .
$$

if and only if it has boundary values equal to $\phi$.

Proof. If $u \in \mathcal{S}^{p}(D)$ has boundary values $\phi$ then (7) holds by Theorem 5.4.

In view of Theorem 6.1 to prove the converse it suffices to show that the potential

$$
v(x)=\int_{D} G_{D}(x, y) \Delta u(y)
$$

has zero boundary value. For this since $v \leq 0$ it suffices to show that $v$ has weak limit values equal to 0 with respect to any sequence $M=\left\{\mu_{r_{j}}\right\}, r_{j} \nearrow 0$.

Let $C \geq f \geq 0$ be a continuous function on $\bar{D}$. Suppose that $r<0$ and $x_{0} \in D_{r}$. Then

$$
f_{r}(y)=\int_{S_{r}} f(x) G_{D}(x, y) d \mu_{r}(x) \geq C \int_{S_{r}} G_{D}(x, y) d \mu_{r}(x) .
$$

Since $G_{D}(x, y)$ is subharmonic in $x$ when $y \in D_{r}$ and harmonic in $x$ on $D_{r}$ when $y \in D \backslash \bar{D}_{r}$,

$$
\int_{S_{r}} G_{D}(x, y) d \mu_{r}(x) \geq G_{D}\left(x_{0}, y\right) .
$$

Moreover, for any $r_{0}<0$ and any $\varepsilon>0$ there is $r_{1}<0$ such that if $r_{1}>r>0$ and $y \in D_{r_{0}}$, then $G_{D}(x, y)>-\varepsilon$ when $x \in D \backslash \bar{D}_{r_{1}}$. Thus $f_{r}(y) \geq-C \varepsilon$ on $D_{r_{0}}$ and $f_{r}(y) \geq-C G_{D}\left(x_{0}, y\right)$ on the rest of the domain. Hence

$$
\int_{S_{r}} f(x) v(x) d \mu_{r}(x)=\int_{D} f_{r}(y) \Delta u(y) \rightarrow 0
$$

as $r \rightarrow 0^{-}$and we see that $v$ has weak limit values equal to 0 with respect to any sequence $M=\left\{\mu_{r_{j}}\right\}, r_{j} \nearrow 0$.

\section{SpaCes of PluRisubharmonic FunCtions}

Let $D$ be a hyperconvex domain in $\mathbb{C}^{n}$, i.e. it has a continuous plurisubharmonic exhausting function $u$ equal to 0 on $\partial D$. We assume that such functions can take $-\infty$ as their value.

On such domains for each $w \in D$ there is a unique continuous plurisubharmonic function $G(z, w)=g_{w}(z)$ on $\bar{D} \times D$ equal to 0 on $\partial D \times D$, satisfying the equation $\left(d d^{c} g_{w}\right)^{n} \equiv 0$ on $D \backslash\{w\}$ and $g_{w}(z)-\log |z-w|=O(1)$ as $z \rightarrow w$. The function $g_{w}$ is called the pluricomplex Green function on $D$ with the pole at $w$. 
For $w \in D$ and $r<0$ we let $g_{w, r}=\max \left\{g_{w}, r\right\}$. Following [D] we let

$$
\mu_{w, r}=\frac{1}{(2 \pi)^{n}}\left(\left(d d^{c} g_{w, r}^{n}-\chi_{D \backslash B_{w, r}}\left(d d^{c} g_{w}\right)^{n}\right),\right.
$$

where the domain $B_{w, r}=\left\{z \in D: g_{w}<r\right\}$. The measure $\mu_{w, r}$ is nonnegative and supported by $S_{w, r}=\left\{z \in D: g_{w}=r\right\}$. As $r \rightarrow 0^{-}$the measures $\mu_{w, r}$ converge weak-* to a measure $\mu_{w}$.

In [D, Chapter 5] Demailly had proved the following Lelong-Jensen formula.

Theorem 7.1. Let $D$ be a hyperconvex domain and $w_{0} \in D$. There is a nonnegative Borel function $P_{D}(w, \zeta)$ on $D \times \partial D$ such that $\mu_{w}(\zeta)=P_{D}(w, \zeta) \mu_{w_{0}}(\zeta)$ and if $u$ is a continuous function on $\bar{D}$ plurisubharmonic on $D$, then

$$
u(w)=\int_{\partial D} u(\zeta) P_{D}(w, \zeta) d \mu_{w_{0}}(\zeta)+\frac{1}{(2 \pi)^{n}} \int_{D} g_{w} d d^{c} u \wedge\left(d d^{c} g_{w}\right)^{n-1} .
$$

In particular, if $u \equiv 1$ we see that

$$
\int_{\partial D} P_{D}(w, \zeta) d \mu(\zeta)=1 .
$$

To expand the space $P S^{c}(D)$ of continuous function on $\bar{D}$ plurisubharmonic on $D$ the following construction is natural (see [PS] for more details). Let us fix a point $w_{0} \in D$ and define the space $P S_{w_{0}}^{p}(D)$ as the space of plurisubharmonic functions $u$ on $D$ such that

$$
\limsup _{r \rightarrow 0^{-}} \int_{S_{w_{0}, r}}|u|^{p} d \mu_{w_{0}, r}<\infty .
$$

We will denote the latter limit as $\|u\|_{P S_{w_{0}}^{p}(D)}$.

The domains $B_{r}=B_{w_{0}, r}$ are also hyperconvex. For $r<0$ we denote by $g_{w, r}(z)=$ $g_{B_{r}}(z, w)$ the pluricomplex Green function on $B_{r}=B_{w_{0}, r}$ and let $\widetilde{\mu}_{w, r}$ be the measure generated by $g_{w, r}$ on $S_{r}=S_{w_{0}, r}$.

Lemma 7.2. For any $w \in D$ the measures $\widetilde{\mu}_{w, r}$ converge weak-* to $\mu_{w}$ as $r \rightarrow 0^{-}$, i.e., $\widetilde{\mu}_{w, r}(u)$ converge to $\mu_{w}(u)$ as $r \rightarrow 0^{-}$when $u \in C(\bar{D})$.

Proof. Since any function in $C(\bar{D})$ can be uniformly approximated by $C^{2}$-functions on $\mathbb{C}^{n}$ which are differences of plurisubharmonic $C^{2}$-functions, it suffices to prove this theorem when $u$ is plurisubharmonic and $C^{2}$ on $\mathbb{C}^{n}$.

By Theorem 5.4

$$
u(w)=\int_{S_{r}} u(\zeta) d \widetilde{\mu}_{w, r}(\zeta)+\frac{1}{(2 \pi)^{n}} \int_{B_{r}} g_{w, r} d d^{c} u \wedge\left(d d^{c} g_{w, r}\right)^{n-1} .
$$

Since the functions $g_{w, r}$ decrease when $r$ increases and converge to $g_{w}$ we see that the volume integrals in the latter formula converge to

$$
\int_{D} g_{w} d d^{c} u \wedge\left(d d^{c} g_{w}\right)^{n-1} .
$$

Hence $\widetilde{\mu}_{w, r}(u)$ converges to $\mu_{w}(u)$ as $r \rightarrow 0^{-}$.

Let $P_{r}=P_{B_{r}}$. Our first step is the following lemma. 
Lemma 7.3. Let $D$ be a hyperconvex domain in $\mathbb{C}^{n}$, let $K$ be a compact set in $D$ and $w_{0} \in K$. There is a constant $c>0$ depending only on $K$ and $D$ such that $c \widetilde{\mu}_{w_{1}, r} \leq \mu_{w_{2}, r}(z) \leq c^{-1} \widetilde{\mu}_{w_{1}, r}$ for any points $w_{1}, w_{2} \in K$ when $r$ is sufficiently close to 0 .

Consequently, the functions $P_{r}(z, w)$ are uniformly bounded on $S_{r}$ when $w \in K$ and $r$ is sufficiently close to 0.

Proof. Let us take $r_{0}$ such $K \subset B_{r_{0}}=B_{w_{0}, r_{0}}$. By the continuity of the pluricomplex Green functions there is a constant $a>0$ such that $-a \leq g_{w} \leq-a^{-1}$ on $S_{r_{0}}$ for any $w$ in $K$. Hence there is a constant $b>0$ such that $b g_{w_{1}} \leq g_{w_{2}} \leq b^{-1} g_{w_{1}}$ for any $w_{1}, w_{2} \in K$. By the maximality of pluricomplex Green functions this inequality holds on $D \backslash B_{r_{0}}$.

The functions $g_{w, r}$ are continuous, decreasing in $r$ and converging to $g_{w}$ pointwise. Hence they converge to $g_{w}$ uniformly on compacta as $r \rightarrow 0^{-}$and we can find $r_{1}$ between $r_{0}$ and 0 such that $g_{w} \leq g_{w, r}<2 g_{w}$ on $S_{r_{0}}$ for all $w \in K$. Thus there is a constant $c>0$ such that $c g_{w_{1}, r} \leq g_{w_{2}, r} \leq c^{-1} g_{w_{1}, r}$ on $S_{r_{0}}$ for any $w_{1}, w_{2} \in K$ when $r$ is sufficiently close to 0 . By the maximality of pluricomplex Green functions this inequality holds on $B_{r} \backslash B_{r_{0}}$. By [D, Theorem 3.8] $c \widetilde{\mu}_{w_{1}, r} \leq \widetilde{\mu}_{w_{2}, r}(z) \leq c^{-1} \widetilde{\mu}_{w_{1}, r}$.

Since $\widetilde{\mu}_{w, r}(z)=P_{r}(w, z) \widetilde{\mu}_{w_{0}, r}$ we see that the functions $P_{r}(z, w)$ are uniformly bounded on $S_{r}$ when $w \in K$.

As the result of two lemmas above we have the following corollary.

Corollary 7.4. Let $D$ be a hyperconvex domain and $w_{0} \in D$. If a function $u \in$ $P S_{w_{0}}^{p}(D), p>1$, has boundary values $u^{*}$, then

$$
u(w)=\int_{\partial D} u^{*}(\zeta) P_{D}(w, \zeta) d \mu_{w_{0}}(\zeta)+\frac{1}{(2 \pi)^{n}} \int_{D} g_{w} d d^{c} u \wedge\left(d d^{c} g_{w}\right)^{n-1} .
$$

Proof. For each $z \in D$ the functions $P_{r}(z, \zeta)$ are non-negative and uniformly bounded on $S_{r}$ when $r$ is close to 0 and the kernel $P(z, \cdot)$ is the weak limit values of the functions $P_{r_{j}}(z, \cdot)$ with respect to any sequence $M=\left\{\mu_{w_{0}, r_{j}}\right\}, r_{j} \nearrow 0$

The equation (8) holds if $D$ is replaced by $D_{r}, r<0$, and $u^{*}$ replaced by $u$. Since the surface integrals in (8) stay bounded and volume integrals are negative and decrease as $r \rightarrow 0^{-}$, by Theorem 5.4 we have (8) on $D$.

Theorem 7.5. Let $D$ be a strongly pseudoconvex domain with $C^{2}$ boundary and $w_{0} \in D$. If a function $u \in P S_{w_{0}}^{p}(D), p>1$, then it has boundary values $u^{*}$.

Proof. Let $\rho$ be a defining function of $D$. It was proved in [D, Theorem 6.1] that there are positive constants $C_{1}$ and $C_{2}$ such that

$$
C_{1}\left(d d^{c} \rho\right)^{n-1} \wedge d^{c} \rho \leq \mu_{w_{0}, r} \leq C_{2}\left(d d^{c} \rho\right)^{n-1} \wedge d^{c} \rho
$$

on $S_{r}$. The form $\left(d d^{c} \rho\right)^{n-1} \wedge d^{c} \rho$ is continuous and strictly positive and, therefore, there are positive constants $A_{1}$ and $A_{2}$ such that $A_{1} \lambda_{r} \leq \mu_{w_{0}, r} \leq A_{2} \lambda_{r}$ on $S_{r}$, where $\lambda_{r}$ is the surface area.

Let $n(\zeta)$ be the inward normal vector at $\zeta \in \partial D$. It follows from above that $\zeta+\operatorname{tn}(\zeta)$ is a radial mapping. Since $u$ is a subharmonic function on $D$ which has a harmonic majorant, by $[\mathrm{S}] u$ has radial limits $\mu_{w_{0}}$-a.e. with respect to $n$. By Theorem $5.5 u$ has boundary values $u^{*}$. 
As an example of a non-smooth domain we can offer only a polydisk. If $D=\mathbb{D}^{n}$, $w=\left(w_{1}, \ldots, w_{n}\right)$ and $z=\left(z_{1}, \ldots, z_{n}\right)$, then (see $\left.[\mathrm{D}]\right)$

$$
g_{w}(z)=\max _{1 \leq j \leq n} \log \left|\frac{z_{j}-w_{j}}{1-\bar{w}_{j} z_{j}}\right| .
$$

Theorem 7.6. Let $D=\mathbb{D}^{n}$ and $w_{0} \in D$. If a function $u \in P S_{w_{0}}^{p}(D), p>1$, then it has boundary values $u^{*}$.

Proof. We will prove it for $w_{0}=0$. Other cases are easily obtained by biholomorphic transformations moving $w_{0}$ to 0 . Since (see $[\mathrm{D}]$ ) $\mu_{r}$ is supported by the Shilov boundary of $\mathbb{D}_{r}^{n}$ and is equal to $(2 \pi)^{-n} d \theta_{1} \ldots d \theta_{n}, z_{j}=r e^{i \theta_{j}}$, we see that if $u \in P S_{0}^{p}(D)$ and $\theta=\left(\theta_{1}, \ldots, \theta_{n}\right)$, then $u \in P S_{0}^{p}\left(\mathbb{D}_{\theta}\right)$, where $\mathbb{D}_{\theta}=\{z=$ $\left.\xi\left(e^{i \theta_{1}}, \ldots, e^{i \theta_{n}}\right), \xi \in \mathbb{D}\right\}$ for $\mu_{0}$-almost all $\theta$.

If the radial mapping is $v(\zeta, t)=(1-t) \zeta$ then $u$ has radial limits $\mu_{0}$-almost all $\theta$ and by Theorem $5.5 u$ has boundary values $u^{*}$.

\section{REFERENCES}

[AG] D. H. Armitage, S. J. Gardiner, Classical Potential Theory, Springer, 2001.

[BPT] F. Bracci, G. Patrizio, S. Trapani, The pluricomplex Poisson kernel for strongly convex domains, Trans. Amer. Math. Soc., 361 (2009), 979-1005.

[CK] U. Cegrell, B. Kemppe, Monge-Ampre boundary measures, Ann. Polon. Math., 96 (2009), 175-196.

[Da] B. E. J. Dahlberg, On the existence of radial boundary values for functions subharmonic in a Lipschitz domain, Indiana Univ. Math. J. 27 (1978), 515-526.

[D] J.-P. Demailly, Mesure de Monge-Ampere et mesures plurisousharmonique, Math. Z., 194(1987), 519-564.

[H] L. L. Helms, Introduction to Potential Theory, John Wiley\&Sons, 1969.

[JK1] D. S. Jerison, C. E. Kenig, The Dirichlet problem in nonsmooth domains, Ann. of Math., 113 (1981), 367-382.

[JK2] D. S. Jerison, C. E. Kenig, Boundary behavior of harmonic functions in nontangentially accessible domains, Adv. in Math., 46 (1982), 80-147.

[PS] E. A. Poletsky, M. I. Stessin, Hardy and Bergman spaces on hyperconvex domains and their composition operators, Indiana Univ. Math. J., 57 (2008), 2153-2201.

[S] E. D. Solomentsev, Boundary values of subharmonic functions, Czech. Math. J., v. 8 (1958), 520-534

Department of Mathematics, 215 Carnegie Hall, Syracuse University, Syracuse, Ny 13244,EAPOLETS@SYR.EDU 